\title{
A Nash-Hörmander iteration and boundary elements for the Molodensky problem
}

\author{
Adrian Costea* Heiko Gimperlein ${ }^{\dagger} \quad$ Ernst P. Stephan*
}

November 20, 2018

\begin{abstract}
We investigate the numerical approximation of the nonlinear Molodensky problem, which reconstructs the surface of the earth from the gravitational potential and the gravity vector. The method, based on a smoothed Nash-Hörmander iteration, solves a sequence of exterior oblique Robin problems and uses a regularization based on a higher-order heat equation to overcome the loss of derivatives in the surface update. In particular, we obtain a quantitative a priori estimate for the error after $m$ steps, justify the use of smoothing operators based on the heat equation, and comment on the accurate evaluation of the Hessian of the gravitational potential on the surface, using a representation in terms of a hypersingular integral. A boundary element method is used to solve the exterior problem. Numerical results compare the error between the approximation and the exact solution in a model problem.
\end{abstract}

Key words: Molodensky problem, single layer potential, second-order derivatives, heatkernel smoothing,

\section{Introduction}

The determination of the shape of the earth and its exterior gravitational field from terrestrial measurements is a basic problem in physical geodesy [1, 2]. It is usually formulated as an exterior free boundary problem for the Laplace equation in $\mathbb{R}^{3}$ with boundary conditions corresponding to the type of observation. In the formulation introduced by Molodensky [3, 4], the gravitational potential $W$ and field $G$ are prescribed on an unknown boundary diffeomorphic to the 2 -sphere by a map $\varphi: S^{2} \rightarrow \mathbb{R}^{3}$. With the advent of satellite technologies to determine the earth's surface, high-precision studies combine satellite data with local gravity measurements.

The mathematical analysis of Molodensky's problem was initiated by Hörmander [5], who investigated the local existence and uniqueness of solutions based on the implicit function theorem for $C^{\infty}$-functions on the boundary. In this article, we consider the mathematically justified numerical analysis of the Molodensky problem, using a feasible reformulation of the constructive

\footnotetext{
*Institute of Applied Mathematics, Leibniz University Hannover, Welfengarten 1, 30167 Hannover, Germany, email: \{costea, stephan\}@ifam.uni-hannover.de

${ }^{\dagger}$ Department of Mathematical Sciences, University of Copenhagen, Universitetsparken 5, 2100 Copenhagen $\varnothing$ Denmark, email: gimperlein@math.ku.dk
} 
proof of existence.

To solve the free boundary problem, we iteratively construct a sequence $\left(\varphi_{m}\right)_{m \in \mathbb{N}_{0}}$ of approximate solutions, where $\varphi_{m}$ is obtained from the solution to the problem linearized around $\varphi_{m-1}$. As the main difficulty, the solution operator to the linearization is of order 2. It is unbounded in the natural Banach spaces of functions, and approximations constructed from Banach fixedpoint iterations will eventually lose regularity. Based on the insights of Nash [6] and Moser [7], Hörmander defines a smoothened, convergent sequence of approximate solutions for data, which are close in a Hölder norm to those of a given solution.

Note that the increments $\varphi_{m+1}-\varphi_{m}$ differ from certain increments considered in the geodesic literature. In our case, the linearized problem involves the solution of an exterior boundary problem for the homogeneous Laplace equation, and no "topographic-isostatic" correction is necessary to correct for unknown masses between $\varphi_{m}\left(S^{2}\right)$ and $\varphi_{0}\left(S^{2}\right)$.

In this work, we show that a variant of this construction is numerically feasible and obtain an a priori estimate for the error in the $m$-th step. We test it on a simple example, in which the algorithm is started with the canonical embedding of the unit sphere in $\mathbb{R}^{3}$ and recovers the sphere of radius 1.1. The reader will find a precise exposition of our main results below. In particular, we legitimize the use of smoothing operators based on the solution of higher-order heat equations in this context. We also numerically explore the accurate evaluation of second-order derivatives on the boundary.

This article shows that the rigorous numerical solution of the nonlinear Molodensky problem is computationally feasible. To improve the stability and numerical accuracy of the method proposed in this paper, further development needs to focus on the domain discretization error, comparing higher-order approximations of the surface with meshless methods for realistic data. In particular, to be relevant for the geodesic community, one might model the exact surface by the ETOPO1 model of the earth and compute the gravity vector from the EGM2008 model. A more realistic model problem could then try to recover $\varphi$ starting from the GRS80 ellipsoid as surface $\varphi_{0}$ and the corresponding Somigliani-Pizetti field as $\left(W_{0}, G_{0}\right)[8]$.

For simplicity, most of this article considers the case of a nonrotating earth. The analysis, however, readily extends to the general case.

\subsection{Related work}

Local existence for the Molodensky problem with data in a neighborhood of a given solution consisting of a gravitational potential $W_{0}$, gravitational field $G_{0}$ and surface $\varphi_{0}$, regularity and uniqueness were first established by Hörmander.

Theorem 1 (Theorem 3.4.1, [5]). Assume that $\left(W_{0}, G_{0}, \varphi_{0}\right)$ satisfies the assumptions in Section 2. and let $\epsilon>0$. 
a) For all $W, G$ in an $\mathscr{H}^{2+\epsilon}$ neighborhood of $W_{0}, G_{0}$, the Molodensky problem admits a solution $\varphi$ close to $\varphi_{0}$ in $\mathscr{H}^{2+\epsilon}$.

b) If $W, G$ are in $\mathscr{H}^{a}$ for some non-integer a $>2+\epsilon$, then $\varphi \in \mathscr{H}^{a}$.

c) Any small $\mathscr{H}^{3+\epsilon}$ neighborhood of $\varphi_{0}$ contains at most one solution of the problem.

See the appendix for our definition of the space $\mathscr{H}^{a}$ of Hölder-continuous functions. Based on ideas of Nash and Moser, Hörmander found an iterative method of solution, which overcomes the loss of derivatives in each iteration step with an abstract smoothing operator. We consider the numerical aspects of his approach and discuss an implementation using the boundary element method (BEM).

The numerical solution of the linearized Molodensky problem using linear boundary elements has first been analyzed by Klees et al. 9], see also work by Holota [10] or Freeden and coauthors [11. Their general convergence analysis implies the convergence and stability of our solution, and we refer to their paper for a discussion of the linearized problem.

To overcome a loss of derivates in simple ordinary differential equations, smoothed iterative solvers have been investigated by Jerome [12] and Jerome and Fasshauer [13]. The smoothing step would usually involve the solution of a heat equation, which does not satisfy the non-saturation property made in previous theoretical analyses (Property 1 (iii) in Section 3). However, smoothing with the help of the heat equation is used in a variety of contexts such as high-dimensional statistics or image processing, and numerous optimized implementations are readily available. Our analysis rigorously legitimizes their application. We are not aware of previous numerical investigations of smoothed fixed-point iterations for 2 - or higher-dimensional problems.

There has been recent interest in the numerical analysis of semilinear elliptic and parabolic equations on the sphere (see e.g. [14]). Our article focuses mostly on the specific difficulties of the Molodensky problem.

\subsection{Formulation of the problem}

In the following, the earth is assumed to be a rigid body of mass $M$, which rotates with a constant and known angular velocity $\omega$ around the $x_{3}$ axis through the center of mass 0 . The surface is diffeomorphic to the sphere $S^{2}=\left\{x \in \mathbb{R}^{3} ; x_{1}^{2}+x_{2}^{2}+x_{3}^{2}=1\right\}$ under a map $\varphi: S^{2} \rightarrow \mathbb{R}^{3}$. The measured data $W$ and $G$ may then be considered as functions on $S^{2}$

$$
W: S^{2} \rightarrow \mathbb{R}, \quad G: S^{2} \rightarrow \mathbb{R}^{3}
$$

The Molodensky problem is to find a sufficiently smooth embedding $\varphi: S^{2} \rightarrow \mathbb{R}^{3}$ such that

$$
W=w \circ \varphi, \quad G=\nabla w \circ \varphi=g \circ \varphi \quad \text { on } \quad S^{2},
$$

where $w: \varphi\left(S^{2}\right) \rightarrow \mathbb{R}$ denotes the gravity potential, $g=\nabla w: \varphi\left(S^{2}\right) \rightarrow \mathbb{R}^{3}$ the gravity vector. The static gravitational potential $v$ is harmonic in the exterior of the earth with boundary values

$$
w(x)=v(x)+\frac{\omega^{2}}{2}\left(x_{1}^{2}+x_{2}^{2}\right) \quad \text { on } \quad \varphi\left(S^{2}\right) .
$$


The center of mass is fixed to 0 by the radiation condition

$$
v(x)=\frac{M}{|x|}+\mathcal{O}\left(|x|^{-3}\right) \quad \text { for } \quad x \rightarrow \infty .
$$

We also require that the Marussi condition [15],

$$
\operatorname{det} g^{\prime}(x) \neq 0, \quad x \in \varphi\left(S^{2}\right)
$$

is fulfilled and set

$$
h=-g^{\prime-1} g .
$$

To determine $\varphi$, we start with a $C^{\infty}$ solution of the Molodensky problem $\left(\varphi_{0}, W_{0}, G_{0}\right)$ such that (4) is satisfied and the corresponding $h_{0}$ is never tangential to $\varphi_{0}\left(S^{2}\right)$. Linearization around $\left(\varphi_{0}, W_{0}, G_{0}\right)$ results in the oblique Robin problem

$$
\Delta u=0 \quad \text { outside } \varphi_{0}\left(S^{2}\right),\left(u+\left\langle\operatorname{grad} u, h_{0}\right\rangle\right) \circ \varphi=f \quad \text { on } \quad S^{2}, u(x)=\frac{M}{|x|}+\mathcal{O}\left(|x|^{-3}\right) .
$$

Under certain conditions on $\varphi$ and $h$, which we assume to hold in what follows, the oblique Robin problem is a regular elliptic boundary value problem, and Fredholm's alternative holds. The oblique Robin problem can be shown to have Fredholm index 0, and the homogeneous problem admits three linearly independent eigensolutions. Existence and uniqueness are therefore assured provided that $f$ belongs to a subspace of $C^{\infty}(\partial \Omega)$ of codimension 3 .

For example, the linearized problem is well-posed when $\varphi_{0}\left(S^{2}\right)$ is the unit sphere and $W_{0}, G_{0}$ are the Newton potential and its gradient. The three-dimensional subspace is then spanned by the spherical harmonics $\left\{Y_{1,-1}, Y_{1,0}, Y_{1,1}\right\}$ of degree 1. A careful analysis in [5], Theorem 1.5.1 and subsequent examples, shows that the problem remains well-posed as long as $\varphi$ is close to the identity and $h$ makes a small angle with both the exterior normal vector field and the radial vector field $\frac{x}{|x|}$. Numerically, the conditions are satisfies e.g. for any topography with slopes $<41^{\circ}$, provided slopes $>10^{\circ}$ are sufficiently rare. In general, we may then use the restrictions $\left\{A_{j}\right\}_{j=1}^{3}$ to $\varphi_{0}\left(S^{2}\right)$ of harmonic functions $u_{j}^{\varphi_{0}}$ with $u_{j}^{\varphi_{0}}(x) \rightarrow 0$ for $|x| \rightarrow \infty$, such that the first degree harmonics in the multipole expansion at infinity are linearly independent.

The augmented formulation of the linearized problem now reads as follows: Find $u$ and constants $a_{j} \in \mathbb{R}$ such that

$$
\begin{aligned}
& \Delta u=0 \quad \text { outside } \quad \varphi\left(S^{2}\right), \\
& u+\nabla u \cdot h=f-\sum_{j=1}^{3} a_{j} A_{j} \quad \text { on } \quad \varphi\left(S^{2}\right) \\
& u(x)=\frac{c}{|x|}+O\left(|x|^{-3}\right) \quad \text { when } \quad|x| \rightarrow \infty, \quad c \in \mathbb{R},
\end{aligned}
$$

for suitable $f \in C^{\infty}\left(\varphi\left(S^{2}\right)\right)$. 
The iterative solution of the Molodensky problem solves a sequence of linearized problems with

$$
f_{m}=\dot{W}_{m} \circ \varphi_{m}^{-1}+\left(\dot{G}_{m} \circ \varphi_{m}^{-1}\right) \cdot h_{m}
$$

as specified in Section 2 and updates $\varphi$ by an increment proportional to $\dot{\varphi}=(\nabla g \circ \varphi)^{-1}(\dot{G}-\nabla u \circ \varphi)$. As the main difficulty of the Molodensky problem, the update $\dot{\varphi}$ is in general less regular than $\varphi$.

\subsection{Error estimates}

We complement Hörmander's existence result by giving an a priori estimate for the error between the solution and the $m$-th iterative approximation. Here and in the remainder of the article, $\epsilon>0$ will be such that the relevant Hölder exponent is not an integer.

A sequence of approximate solutions $\left(W_{m}, \varphi_{m}\right)$ will be defined in Section 2, depending on a given solution $\left(W_{0}, G_{0}, \varphi_{0}\right)$ and two parameters $\theta_{0}$ and $\kappa$. Under the assumptions of this section we obtain the following a priori estimate in Hölder norms for data $(W, G)$ in an $\mathcal{H}^{2+\epsilon}$ neighborhood of $\left(W_{0}, G_{0}\right)$ :

Theorem 2. Let $\alpha>2+2 \epsilon, 0<a<\alpha, E=a-\alpha-1$ and $\tau>0$ sufficiently small. Then there exist constants $\theta_{0}, \kappa_{0}>0$ and $C_{\tau}>0$ such that the approximate solutions $\left(W_{m}, \varphi_{m}\right)$ satisfy for all $m \geq 0$ and $\kappa \geq \kappa_{0}$

$$
\left\|W-W_{m}\right\|_{a+\epsilon}+\left\|\varphi-\varphi_{m}\right\|_{a+\epsilon} \leq C_{\tau}\left(\left\|W-W_{0}\right\|_{\alpha+\epsilon}+\left\|G-G_{0}\right\|_{\alpha+\epsilon}\right)\left(\theta_{0}^{\kappa}+m\right)^{\frac{E+1+\tau}{\kappa}}
$$

The constants depend only on the data.

Below, we note that the estimate on $W-W_{m}$ and $\varphi-\varphi_{m}$ implies a corresponding estimate on $G-G_{m}$.

The explicit dependence on the parameters $\theta_{0}$ and $\kappa$ obtained in Theorem 2 allows us to investigate also a restarted algorithm, which is less susceptible to the spreading of numerical errors (see Algorithm 2):

(0) Choose an approximate solution $\left(W_{0}, G_{0}, \varphi_{0}\right)$ and $\theta_{0}$.

(1) Using $\left(W_{0}, G_{0}, \phi_{0}\right)$ do $k$ steps of Hörmander's method leading to $\left(W_{k}, G_{k}, \varphi_{k}\right)$.

(2) Set $\left(W_{0}, G_{0}, \phi_{0}\right)=\left(W_{k}, G_{k}, \varphi_{k}\right)$, a corresponding new $\theta_{0}$ and go to (1).

We denote the approximate solution after $l$ iterations of $(1)$ by $\left(W^{(l)}, G^{(l)}, \varphi^{(l)}\right)$ and the corresponding $\theta_{0}$ by $\theta_{0, l}$. From the Lipschitz-continuity of the map $\Gamma:(W, \varphi) \mapsto G$,

$$
\left\|G-G^{(l-1)}\right\|_{\alpha+\epsilon} \leq C_{K}\left(\left\|W-W^{(l-1)}\right\|_{\alpha+2+\epsilon}+\left\|\varphi-\varphi^{(l-1)}\right\|_{\alpha+2+\epsilon}\right)
$$

on bounded subsets $\left\|\varphi-\varphi_{0}\right\|_{\alpha+2+\epsilon} \leq K$, we obtain

$$
\begin{aligned}
\left\|W-W^{(l)}\right\|_{a+\epsilon}+\left\|\varphi-\varphi^{(l)}\right\|_{a+\epsilon} & \leq C_{\tau}\left(\left\|W-W^{(l-1)}\right\|_{\alpha+\epsilon}+\left\|G-G^{(l-1)}\right\|_{\alpha+\epsilon}\right)\left(\theta_{0, l-1}^{\kappa}+k\right)^{\frac{E+1+\tau}{\kappa}} \\
& \leq C_{\tau}\left(\left\|W-W^{(l-1)}\right\|_{\alpha+\epsilon}+\left\|G-G^{(l-1)}\right\|_{\alpha+\epsilon}\right)\left(\theta_{0, l-1}^{\kappa}+k\right)^{\frac{E+1+\tau}{\kappa}} \\
& \lesssim\left(\left\|W-W^{(l-1)}\right\|_{\alpha+2+\epsilon}+\left\|\varphi-\varphi^{(l-1)}\right\|_{\alpha+2+\epsilon}\right)\left(\theta_{0, l-1}^{\kappa}+k\right)^{\frac{E+1+\tau}{\kappa}}
\end{aligned}
$$


for any $\tau>0$.

Iterating this estimate yields that the sequence of iterates of the restarted algorithm converges, if we choose a sufficiently rapidly increasing sequence of $\theta_{0, l}$. As the proof will show, $\theta_{0, l}$ has to be chosen as a function of certain higher Hölder norms of $W-W^{(l)}$ and $\varphi-\varphi^{(l)}$. Summing up:

Proposition 1. Under the assumptions of the theorem,

$$
\left\|W-W^{(l)}\right\|_{a+\epsilon}+\left\|\varphi-\varphi^{(l)}\right\|_{a+\epsilon} \lesssim\left(\left\|W-W^{(l-1)}\right\|_{\alpha+2+\epsilon}+\left\|\varphi-\varphi^{(l-1)}\right\|_{\alpha+2+\epsilon}\right)\left(\theta_{0, l-1}^{\kappa}+k\right)^{\frac{E+1+\tau}{\kappa}} .
$$

In particular, the algorithm with restart converges for appropriate $\theta_{0, l}$ depending on $W-W_{0}$ and $\varphi-\varphi_{0}$.

Iterating (9), we can estimate $W-W^{(l)}$ and $\varphi-\varphi^{(l)}$ in terms of the initial error $W-W_{0}, \varphi-\varphi_{0}$.

The proofs of Theorem 2 and Proposition 1 are contained in the appendix, Section 6.1 .

\subsection{Numerical considerations}

From a computational perspective, we give a proof-of-principle for fully nonlinear numerical computations with the Molodensky problem. Besides showing the practicality of standard heat equation smoothing, the implementation involves subproblems of possible interest beyond the particular problem:

a) The updates of $\varphi$ and $h$ require the highly accurate evaluation on the surface for first and second derivatives of the gravitational field, expressed as derivatives of the single layer potential [16, 1, 17. We show that second order finite elements are necessary. If we combine them with a partially analytic evaluation of the singular integrals for the gradient and finite differences for the Hessian, the error becomes negligible.

b) Surface discretization turns out to be the dominant source of error for smooth data. This shows the need for third or higher-order approximations or meshless methods. See e.g. [18], Ch. 8, for boundary integral equations on surfaces approximated to degree $p$. The approximately spherical geometry suggests meshless eigenfunction expansions, and [19] contains a first study of in the case of a particular linearized problem.

Parallelization and nontrivial optimization are necessary to deal with the large, dense matrices of a higher-order boundary element formulation and the large number of data points available from geodesic measurements. For the linearized Molodensky problem, panel clustering and fast multipole methods have been discussed in [9], particularly in the case of a non-oblique Robin problem. 


\section{Iterative solution of the nonlinear problem}

Our approach to the Molodensky problem is based on the abstract Nash-Hörmander iteration [5], as adapted to the Molodensky problem. It solves a sequence of linearized problems, whose solutions are less regular than the data, and recovers regularity with the help of a smoothing operator. To simplify notation, we restrict ourselves to a nonrotating earth, $\omega=0$. The equivalence of the formulation to the standard Nash-Moser iteration is discussed in Appendix 6.1. See also 20] for a detailed derivation.

We fix a $C^{\infty}$ solution of the Molodensky problem $\left(\varphi_{0}, W_{0}, G_{0}\right)$ with a gravitational potential $v_{0}$ which satisfies the Marussi condition. We assume that the corresponding $h_{0}$ is not tangential to $\varphi_{0}\left(S^{2}\right)$ and that the homogeneous linearized problem 1.2 has only the trivial solution.

As stated in the introduction, the linearized Molodensky problem involves an oblique Robin problem in the exterior $\mathbb{R}^{3} \backslash \bar{\Omega}_{m}$ of $\varphi_{m}\left(S^{2}\right)$. In each iteration step $m$, we solve the augmented problem:

Given $\dot{W}_{m}: S^{2} \rightarrow \mathbb{R}, \dot{G}_{m}: S^{2} \rightarrow \mathbb{R}^{3}, h_{m}: \varphi_{m}\left(S^{2}\right) \rightarrow \mathbb{R}^{3} \quad$ and $\quad \varphi_{m}: S^{2} \rightarrow \varphi_{m}\left(S^{2}\right) \subset \mathbb{R}^{3}$, find $u_{m}: \mathbb{R}^{3} \backslash \bar{\Omega}_{m} \rightarrow \mathbb{R}$ and constants $a_{j, m} \in \mathbb{R}$ such that

$$
\begin{aligned}
& \Delta u_{m}=0 \quad \text { in } \quad \mathbb{R}^{3} \backslash \bar{\Omega}_{m}, \\
& u_{m}+\nabla u_{m} \cdot h_{m}=f_{m}-\sum_{j=1}^{3} a_{j, m} \widetilde{A}_{j}(x) \quad \text { on } \quad \varphi_{m}\left(S^{2}\right) \\
& u_{m}(x)=\frac{c}{|x|}+O\left(|x|^{-3}\right) \quad \text { when } \quad|x| \rightarrow \infty, \quad c \in \mathbb{R} .
\end{aligned}
$$

Again $f_{m}=\dot{W}_{m} \circ \varphi_{m}^{-1}+\left(\dot{G}_{m} \circ \varphi_{m}^{-1}\right) \cdot h_{m}$. A feasible choice for $\widetilde{A}_{j}$ is discussed after 18.

The solution $u_{m}$ determines a nonlinear correction to $\varphi_{m}, \dot{\varphi}_{m}$, given by

$$
\dot{\varphi}_{m}=\left(\nabla g_{m} \circ \varphi_{m}\right)^{-1}\left(\dot{G}_{m}-\nabla u_{m} \circ \varphi_{m}\right) .
$$

The gravitational potential $g_{m}$ is determined from the approximation to the potential as computed in the first $m$ steps,

$$
\begin{aligned}
& w_{m}=W_{m-1} \circ \varphi_{m}^{-1}+\triangle_{m} u_{m} \quad \text { on } \quad \varphi_{m}\left(S^{2}\right), \\
& W_{m-1}=\left\{\begin{array}{l}
v_{0} \circ \varphi_{0}+\triangle_{0} u_{0} \circ \varphi_{0}+\triangle_{1} u_{1} \circ \varphi_{1}+\cdots+\triangle_{m-1} u_{m-1} \circ \varphi_{m-1}, \quad \text { for } m \geq 1 \\
v_{0} \circ \varphi_{0} \quad \text { for } m=0
\end{array},\right.
\end{aligned}
$$

for suitable stepsizes $\Delta_{j}$ and initial approximation $v_{0}$ by solving an exterior Dirichlet problem: For given $w_{m}$ on $\varphi_{m}\left(S^{2}\right)$, find $\bar{v}_{m}: \mathbb{R}^{3} \backslash \bar{\Omega}_{m} \rightarrow \mathbb{R}$ and constants $a_{j, m} \in \mathbb{R}$ such that

$$
\begin{aligned}
& \Delta \bar{v}_{m}=0 \quad \text { in } \quad \mathbb{R}^{3} \backslash \bar{\Omega}_{m}, \\
& \bar{v}_{\left.m\right|_{\partial \Omega_{m}}}=w_{m}-\left.\sum_{j=1}^{3} a_{j, m} \widetilde{A}_{j}(x)\right|_{x \in \varphi_{m}\left(S^{2}\right)} \text { on } \varphi_{m}\left(S^{2}\right) \\
& \bar{v}_{m}(x)=\frac{c}{|x|}+O\left(|x|^{-3}\right) \quad \text { when } \quad|x| \rightarrow \infty .
\end{aligned}
$$


Here $\widetilde{A}_{j}$ need not be the same as in 10 . For the numerical solution using boundary elements, we can reuse the matrix entries of the discretized Robin problem for this Dirichlet problem. Now equation (11) yields the surface update $\dot{\varphi}_{m}$ to $\varphi_{m}$ using $g_{m}=\nabla \bar{v}_{m}$ and $\nabla g_{m}=\nabla^{2} \bar{v}_{m}$.

The full iterative method involves smoothing in each step based on the solution operator $S_{\theta}$ to a higher-order heat equation as discussed in Section 3. It reads as follows:

\section{Algorithm 1. (Nash-Hörmander algorithm)}

1. For given measured data $W, G$, choose $W_{0}, G_{0}, h_{0}, \varphi_{0}, \theta_{0} \gg 1, \kappa \gg 1$

2. For $m=0,1,2, \ldots$ do

(a) Compute

$$
\theta_{m}=\left(\theta_{0}^{\kappa}+m\right)^{1 / \kappa}, \quad \triangle_{m}=\theta_{m+1}-\theta_{m}
$$

(b) Compute

$$
\begin{aligned}
& \dot{\widetilde{W}}_{0}:=S_{\theta_{0}} \dot{W}_{0}=S_{\theta_{0}}\left(\frac{W-W_{0}}{\triangle_{0}}\right) \\
& \dot{\widetilde{W}}_{m}:=\frac{1}{\triangle_{m}}\left(S_{\theta_{m}}\left(W-W_{0}\right)-S_{\theta_{m-1}}\left(W-W_{0}\right)\right)
\end{aligned}
$$

(c) Compute

$$
\begin{aligned}
\dot{\widetilde{G}}_{0} & :=S_{\theta_{0}} \dot{G}_{0}=S_{\theta_{0}}\left(\frac{G-G_{0}}{\triangle_{0}}\right) \\
\dot{\widetilde{G}}_{m}: & =\frac{1}{\triangle_{m}}\left(S_{\theta_{m}}\left(G-G_{m}+\sum_{j=0}^{m-1} \triangle_{j} \dot{\widetilde{G}}_{j}\right)-S_{\theta_{m-1}}\left(G-G_{m-1}+\sum_{j=0}^{m-2} \triangle_{j} \dot{\widetilde{G}}_{j}\right)\right)
\end{aligned}
$$

(d) Find $u_{m}$ by solving the linearized problem (10) with $\left(\dot{W}_{m}, \dot{G}_{m}\right)$ replaced by $\left(\dot{\widetilde{W}}_{m}, \dot{\widetilde{G}}_{m}\right)$

(e) Find $\bar{v}_{m}$ by solving 13) with $w_{m}$ as defined in 12)

(f) Compute $g_{m}=\nabla \bar{v}_{m}$ and $\nabla g_{m}=\nabla^{2} \bar{v}_{m}$

(g) Compute the surface increment $\dot{\varphi}_{m}$ by

$$
\dot{\varphi}_{m}=\left(\nabla g_{m} \circ \varphi_{m}\right)^{-1}\left(\dot{\widetilde{G}}_{m}-\nabla u_{m} \circ \varphi_{m}\right)
$$

and update surface map by $\varphi_{m+1}=\varphi_{m}+\triangle_{m} \dot{\varphi}_{m}$

(h) Update direction vector and gravity potential by

$$
\begin{aligned}
h_{m+1} & =\left(\left(-\left(\nabla g_{m}\right)^{-1} g_{m}\right) \circ \varphi_{m}\right) \circ\left(\varphi_{m+1}\right)^{-1} \\
G_{m+1} & =g_{m} \circ \varphi_{m}
\end{aligned}
$$

(i) Stop if $\left\|g_{m} \circ \varphi_{m}-G\right\|+\left\|\bar{v}_{m} \circ \varphi_{m}-W\right\|<$ tol 
$\|\cdot\|$ might usually be chosen to be e.g. an $\mathcal{H}^{a}{ }_{-}$norm.

We also consider a variant of the algorithm which is restarted every $k$ steps, using as initial condition the approximate solution from the $k$-th step:

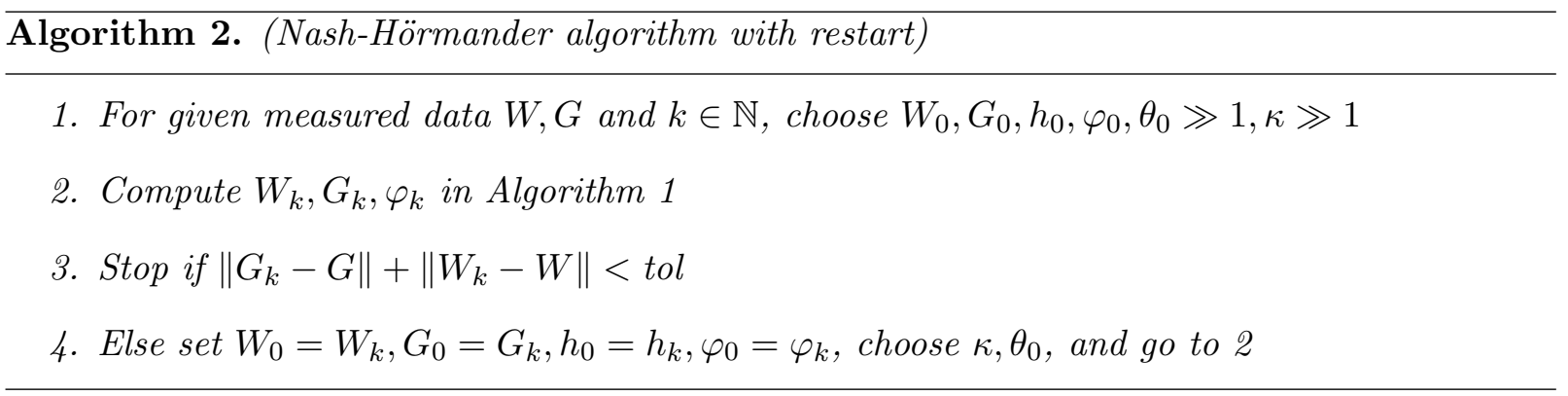

To solve the homogeneous exterior Robin and Dirichlet problems (10) resp. (13), we use a single layer potential ansatz for $u_{m}=V_{m} \mu_{m}$ and satisfy the decay condition at $\infty$ in a weak sense. Boundary element formulations for the oblique Robin problem 10 with general boundary data were first analyzed in [9]. They, in particular, showed the relevance of multipole expansions and panel clustering for the fast solution for experimental geodesic data.

The ansatz transforms 10 into a saddle point problem for the integral operator

$$
\mathcal{S}=V+\frac{1}{2} \cos (\measuredangle(\boldsymbol{n}, \boldsymbol{h})) I+K^{\prime}(\boldsymbol{h}),
$$

on $\varphi_{m}\left(S^{2}\right)$, which is defined in terms of the multilayer potentials

$$
V \mu(x)=\int_{\Gamma} \frac{\mu(y)}{4 \pi|x-y|} d s_{y}, \quad K^{\prime}(\boldsymbol{h}) \mu(x)=\boldsymbol{h} \cdot \nabla \int_{\Gamma} \frac{\mu(y)}{4 \pi|x-y|} d s_{y} .
$$

In particular for $\boldsymbol{h}=\boldsymbol{n}$ the unit normal vector, $K^{\prime}(\boldsymbol{n})$ is the standard adjoint double layer potential.

Solving the Robin problem for arbitrary $\dot{W}, \dot{G}$ requires an appropriate choice of $\widetilde{A}_{j}$. Let $A_{j}=\frac{x_{j}}{|x|^{3}}$ and $\mathcal{N}:=\operatorname{span}\left\{A_{j}\right\}_{j=1, \ldots, 3}$. Then $u_{m}=V_{m} \mu_{m}$ satisfies the decay condition of 10 , whenever $\mu_{m} \in L^{2}\left(\varphi_{m}\left(S^{2}\right)\right) \cap \mathcal{N}^{\perp}$. Since $f_{m}=\dot{W}_{m} \circ \varphi_{m}^{-1}+\left(\dot{G}_{m} \circ \varphi_{m}^{-1}\right) \cdot h_{m} \in L^{2}\left(\varphi_{m}\left(S^{2}\right)\right)$, but not necessarily in $S\left(L^{2}\left(\varphi_{m}\left(S^{2}\right)\right) \cap \mathcal{N}^{\perp}\right), \widetilde{A}_{j}$ must be chosen such that $\operatorname{span}\left\{\widetilde{A}_{j}\right\}_{j=1}^{3}+S\left(L^{2}\left(\varphi_{m}\left(S^{2}\right)\right) \cap\right.$ $\left.\mathcal{N}^{\perp}\right)=L^{2}\left(\varphi_{m}\left(S^{2}\right)\right)$ for 10 to be well defined. $\widetilde{A}_{j}:=\left.S A_{j}\right|_{\varphi_{m}\left(S^{2}\right)}$ is a feasible choice and leads to an equivalent variational formulation of $(10)$ :

Find $\left(\mu_{m}, a_{m}\right) \in L^{2}\left(\varphi_{m}\left(S^{2}\right)\right) \times \mathbb{R}^{3}$ such that

$$
\begin{array}{ccrl}
\left\langle\mathcal{S} \mu_{m}, \phi\right\rangle_{\varphi_{m}\left(S^{2}\right)}+\left\langle\mathcal{S} \sum_{j=1}^{3} a_{j, m} A_{j}, \phi\right\rangle_{\varphi_{m}\left(S^{2}\right)} & =\left\langle f_{m}, \phi\right\rangle_{\varphi_{m}\left(S^{2}\right)} & & \forall \phi \in L^{2}\left(\varphi_{m}\left(S^{2}\right)\right) \\
\left\langle\mu_{m}, A_{k}\right\rangle_{\varphi_{m}\left(S^{2}\right)} & =0 & & \forall k \in\{1,2,3\} .
\end{array}
$$


Note that any $L^{2}$-solution of the weak problem is actually $C^{\infty}$. Indeed, $\mathcal{S}$ is an elliptic pseudodifferential operator, so that $\mu_{m} \in L^{2}$ and $\mathcal{S} \mu_{m}=f_{m}-\mathcal{S} \sum_{j=1}^{3} a_{j, m} A_{j} \in C^{\infty}$ in the distributional sense implies $\mu_{m} \in C^{\infty}[21]$.

The Dirichlet problem 13 is similarly reformulated: Find $\left(\tilde{\mu}_{m}, \widetilde{a}_{m}\right) \in H^{-1 / 2}\left(\varphi_{m}\left(S^{2}\right)\right) \times \mathbb{R}^{3}$ such that

$$
\begin{aligned}
& \left\langle V \tilde{\mu}_{m}, \xi\right\rangle_{\varphi_{m}\left(S^{2}\right)}+\left\langle V \sum_{j=1}^{3} \widetilde{a}_{j, m} A_{j}, \xi\right\rangle_{\varphi_{m}\left(S^{2}\right)}=\left\langle w_{m}, \xi\right\rangle_{\varphi_{m}\left(S^{2}\right)} \forall \xi \in H^{-1 / 2}\left(\varphi_{m}\left(S^{2}\right)\right) \\
& \left\langle\tilde{\mu}_{m}, A_{k}\right\rangle_{\varphi_{m}\left(S^{2}\right)}=0 \quad \forall k \in\{1,2,3\} .
\end{aligned}
$$

On the right hand side,

$$
\begin{aligned}
\left\langle w_{m}, \xi\right\rangle_{\varphi_{m}\left(S^{2}\right)}= & \left\langle W_{m-1} \circ \varphi_{m}^{-1}, \xi\right\rangle_{\varphi_{m}\left(S^{2}\right)}+\triangle_{m}\left\langle u_{m}, \xi\right\rangle_{\varphi_{m}\left(S^{2}\right)} \\
= & \left\langle\left(v_{0} \circ \varphi_{0}\right) \circ \varphi_{m}^{-1}, \xi\right\rangle_{\varphi_{m}\left(S^{2}\right)}+\sum_{i=0}^{m-1} \triangle_{i}\left\langle\left(V_{i} \mu_{i} \circ \varphi_{i}\right) \circ \varphi_{m}^{-1}, \xi\right\rangle_{\varphi_{m}\left(S^{2}\right)} \\
& +\triangle_{m}\left\langle V_{m} \mu_{m}, \xi\right\rangle_{\varphi_{m}\left(S^{2}\right)} .
\end{aligned}
$$

As discussed in Section 4, we use standard boundary element methods for the efficient numerical solution of $(19)$ and 130 . Note that the matrix elements of $V$ have already been computed for the Robin problem.

\section{Smoothing operator}

The smoothing operators $S_{\theta}$ appearing in the iterative solution of the Molodensky problem compensate for the loss of derivatives in the increments of $\varphi$. For a theoretical analysis, smoothing operators $S_{\theta}$ defined from compactly supported functions $\phi \in C_{0}^{\infty}(\mathbb{R})$ are most convenient. If $M$ is a submanifold of $\mathbb{R}^{3}$, we define $S_{\theta}^{t h}: \mathscr{H}^{a}(M) \rightarrow \mathscr{H}^{a}(M)$ as

$$
S_{\theta}^{t h} u:=\phi\left(\frac{1}{\theta^{2}} \Delta_{M}\right) u
$$

Here $\Delta_{M}$ is the Laplace-Beltrami operator associated to the metric which $M$ inherits from $\mathbb{R}^{3}$, and $\phi\left(\frac{1}{\theta^{2}} \Delta_{M}\right) u$ can be computed by expanding $u$ into $L^{2}(M)$-eigenfunctions of $\Delta_{M}$.

These operators have the following properties (Theorem A.10, [5]):

Properties 1. For all $u \in C^{\infty}(M)$ we have

(0) $\left\|S_{\theta}^{t h} u-u\right\|_{a} \stackrel{\theta \rightarrow \infty}{\longrightarrow} 0$;

(i) $\left\|S_{\theta}^{t h} u\right\|_{b} \leq C\|u\|_{a}, \quad b \leq a ;$

(ii) $\left\|S_{\theta}^{t h} u\right\|_{b} \leq C \theta^{b-a}\|u\|_{a}, \quad a \leq b$;

(iii) $\left\|u-S_{\theta}^{t h} u\right\|_{b} \leq C \theta^{b-a}\|u\|_{a}, \quad b \leq a$; 
(iv) $\left\|\frac{d}{d \theta} S_{\theta}^{t h} u\right\|_{b} \leq C \theta^{b-a-1}\|u\|_{a}$

The oscillatory nature of the corresponding integral kernels renders a stable implementation of these operators difficult. In practice, solution operators to heat-like equations are frequently used as smoothing operators in a variety of contexts such as high-dimensional statistics, image processing etc. with readily available, optimized implementations. They have been also used in the numerical explorations of smoothed Newton and similar methods, though without much justification, see e.g [13, 12].

More generally than the heat equation, we consider smoothing operators associated to $\phi(x)=$ $e^{-|x|^{k}}, k \in \mathbb{N}$. They correspond to the time $-1 / \theta^{2 k}$ solution of the $k$-harmonic heat equation

$$
\begin{aligned}
\frac{d}{d t} v(x, t)-A v(x, t) & =0 \quad \text { in } \quad M \times(0, \infty) \\
v(x, 0) & =u(x) \text { in } \quad M
\end{aligned}
$$

with $A:=(-1)^{k+1} \Delta_{M}^{k}$ and $u \in \mathscr{H}^{a}$. Considering $A: \mathscr{H}^{a+2 k} \subset \mathscr{H}^{a} \rightarrow \mathscr{H}^{a}$ as an unbounded operator on the Hölder spaces $(a>0, a \notin \mathbb{N})$ we have the following theorem.

Theorem 3. A generates an analytic semigroup $e^{t A}$ on $\mathscr{H}^{a}$, and the operator $S_{\theta}:=e^{\frac{1}{\theta^{2 k}} A}$ satisfies the properties (0), (i), (ii), (iv) (with $S_{\theta}^{\text {th }}$ replaced by $S_{\theta}$ ) and in addition

$$
\left(i i i^{\prime}\right) \quad\left\|u-S_{\theta} u\right\|_{b} \leq C \theta^{b-a}\|u\|_{a}, \quad \forall 0 \leq a-b<2 k .
$$

A proof of this theorem will be given in the Appendix. In particular, we note that these restricted properties are sufficient to rigorously analyze the convergence of the algorithms presented in Section 2.

\section{Numerical methods}

The main effort to numerically solve the Molodensky problem consists in the accurate solution of the saddle point problems (19) and 20) for the multilayer potentials $\mathcal{S}$ and $V$ on the surfaces $\varphi_{m}\left(S^{2}\right)$.

For the solution, we choose a quasi-uniform triangulation $\mathcal{T}_{h}^{m}$ of $\varphi_{m}\left(S^{2}\right)$ by plane triangles of diameter $\sim h$ and solve the saddle point problems for piecewise polynomial elements on the resulting discretized surface.

In the case of (19), the discretized formulation for the subspace

$$
S_{h, m}^{p}=\left\{\text { space of discontinuous piecewise polynomials of degree } p \text { on } \mathcal{T}_{h}^{m}\right\}=\operatorname{span}\left\{b_{j}\right\}_{j=1}^{N}
$$

of $L^{2}\left(\mathcal{T}_{h}^{m}\right)$ reads as follows: Find $\left(\mu_{m, h}, a_{m, h}\right) \in S_{h, m}^{p} \times \mathbb{R}^{3}$ such that

$$
\begin{gathered}
\left\langle\mathcal{S} \mu_{m, h}, \phi_{h}\right\rangle_{\mathcal{T}_{h}^{m}}+\sum_{j=1}^{3} a_{j, m, h}\left\langle\mathcal{S} A_{j}, \phi_{h}\right\rangle_{\mathcal{T}_{h}^{m}}=\left\langle f_{m}, \phi_{h}\right\rangle_{\mathcal{T}_{h}^{m}} \quad \forall \phi_{h} \in S_{h, m}^{p} \\
\left\langle\mu_{m, h}, A_{k}\right\rangle_{\mathcal{T}_{h}^{m}}=0 \quad \forall k \in\{1,2,3\}
\end{gathered}
$$


Expanding $\mu_{m, h}$ and $\phi_{m, h}$ in the basis $b_{j}$,

$$
\mu_{m, h}=\sum_{j=1}^{N} \mu_{j, m} b_{j}, \quad \phi_{m, h}=\sum_{j=1}^{N} \phi_{j, m} b_{j},
$$

we obtain a matrix equation of the form:

$$
\left[\begin{array}{ll}
\mathcal{S} & \widetilde{\mathcal{S}} \\
\Lambda & 0
\end{array}\right]\left[\begin{array}{l}
\vec{\mu}_{h} \\
\vec{a}_{h}
\end{array}\right]=\left[\begin{array}{l}
\vec{f} \\
\overrightarrow{0}
\end{array}\right]
$$

where $\left(\mathcal{S}_{k j}\right)=\left\langle\mathcal{S} b_{j}, b_{k}\right\rangle,\left(\widetilde{\mathcal{S}_{k j}}\right)=\left\langle\mathcal{S} A_{j}, b_{k}\right\rangle,\left(\Lambda_{k j}\right)=\left\langle b_{j}, A_{k}\right\rangle,\left(f_{k}\right)=\left\langle f_{m}, b_{k}\right\rangle$ and $\vec{\mu} \in \mathbb{R}^{N}, \vec{a} \in \mathbb{R}^{3}$.

For the Dirichlet problem $\sqrt{13}$ we consider $S_{h, m}^{p}$ as a subspace of $H^{-1 / 2}\left(\mathcal{T}_{h}^{m}\right)$, leading to the problem: Find $\left(\tilde{\mu}_{m, h}, \widetilde{a}_{m, h}\right) \in S_{h, m}^{p} \times \mathbb{R}^{3}$ such that

$$
\begin{array}{rlr}
\left\langle V \tilde{\mu}_{m, h}, \xi_{h}\right\rangle_{\mathcal{T}_{h}^{m}}+\sum_{j=1}^{3} \widetilde{a}_{j, m, h}\left\langle V A_{j}, \xi_{h}\right\rangle_{\mathcal{T}_{h}^{m}} & =\left\langle w_{m}, \xi_{h}\right\rangle_{\mathcal{T}_{h}^{m}} & \forall \xi_{h} \in \mathcal{T}_{h}^{m} \\
\left\langle\tilde{\mu}_{m, h}, A_{k}\right\rangle_{\mathcal{T}_{h}^{m}} & =0 \quad \forall k \in\{1,2,3\} .
\end{array}
$$

In matricial form:

$$
\left[\begin{array}{cc}
V & \widetilde{V} \\
\Lambda & 0
\end{array}\right]\left[\begin{array}{l}
\overrightarrow{\widetilde{\mu}}_{h} \\
\overrightarrow{\widetilde{a}}_{h}
\end{array}\right]=\left[\begin{array}{c}
\vec{w}_{m} \\
\overrightarrow{0}
\end{array}\right]
$$

The suggested numerical solution of the linearized Molodensky and Dirichlet problems is stably convergent with quasi-optimal convergence estimates. They are derived in [9, 20] by verifying an inf-sup condition for the boundary element spaces.

Our theoretical analysis legitimizes using the solution of heat-like equations on $\varphi_{m}\left(S^{2}\right)$ as smoothing operator $S_{\theta}$ in the algorithm. We use the implementation presented in [22], which computes an FEM-solution of the heat equation for the geometric Laplace-Beltrami operator by spectral methods. $S_{\theta} F$ is defined as the solution at time $\theta^{-2}$ with initial condition $F$.

\subsection{Details}

The initial sphere $S^{2}$ is triangulated by starting with an icosahedral mesh and subsequent refinements 23]. Assigning to each node a vector in $\mathbb{R}^{3}$ corresponds to a continuous, piecewise linear representation of $\varphi_{m}$. As we need to evaluate second derivatives of the gravitational potential, the polynomial degree on each triangle is $p=2$, and $h_{m}$ is represented by a discontinuous piecewise constant function interpolating the $h_{m}$ from equation 10 in the midpoints of each triangle. Furthermore, $G_{m}$ is the linear interpolation of $\left.g\right|_{\varphi_{m}\left(S^{2}\right)}$, obtained from equation (13), in the nodes. The local basis functions $b_{j}$ are monomials for both the linearized Molodensky problem and for the auxiliary Dirichlet problem. We use analytic expressions to compute $\left\langle V b_{j}, b_{k}\right\rangle$ and $K(h) b_{k}$ ([24]) and perform an hp-composite Gauss quadrature with geometrically graded meshes [25, 17] to determine $\left\langle K^{\prime}(h) b_{j}, b_{k}\right\rangle=\left\langle b_{j}, K(h) b_{k}\right\rangle .\left\langle V A_{j}, b_{k}\right\rangle$ and $\left\langle K^{\prime}(h) A_{j}, b_{k}\right\rangle$ are treated analogously. 
The update of $\varphi$ requires the delicate computation of the Hessian for the gravitational potential, $\nabla g_{m}$, on $\varphi_{m}\left(S^{2}\right)$. Our numerical experiments suggest to derive the component of $g$ in some direction $e$ using the adjoint of the double layer potential, $e \cdot g_{m}=\frac{e \cdot \nu}{2} \mu_{m, h}+K^{\prime}(e) \mu_{m, h}$, and to use finite differences to compute $\nabla g_{m}$. More precisely, for the tangential components we use a central

finite difference scheme, $U^{\prime}(x) \approx \frac{U(x+\delta)-U(x-\delta)}{2 \delta}$, in the exterior domain and extrapolate to the boundary. If $x^{\prime}$ is the point closest to $x$ on the discretized boundary, we choose $\delta$ smaller than the distance of $x^{\prime}$ to the nearest vertex of the triangulation. For the normal component we combine the central finite difference scheme with a Crank-Nicolson method, $U^{\prime}(x) \approx \frac{4 U(x+\delta)-3 U(x)-U(x+2 \delta)}{2 \delta}$, which is forward oriented. The error in both schemes is of order $\delta^{2}$.

Alternative approaches are discussed in detail in Section 5.1.

\section{$5 \quad$ Numerical experiments}

The numerical experiments were carried out on a cluster with 5 nodes à 8 cores with $2.93 \mathrm{Ghz}$ and 48GB memory, where each core uses two Intel Nehalem X5570 processors. With parallelization and optimization we need 20 minutes for each iterations in the case of icosahedron refinements corresponding to 320 triangles $(N=2)$, whereas we need 3 hours for refinements corresponding to 1280 triangles $(N=3)$. A further global refinement would lead to 5120 triangles and 16 times larger dense matrices. For realistic models of the gravity field used in geodesy, the data is often given at grid points spaced less than 10 degrees apart, corresponding e.g. in [9] to triangulations with between 2048 and 131072 triangles. This shows the need to develop local adaptive refinements and matrix compression. Local refinements can be introduced into the restarted Algorithm 2 every $k$ steps.

\subsection{Computation of second derivatives}

The accurate determination of second derivatives of the gravitational potential on the surface is crucial for our approach and is of interest also in other contexts. The first derivatives of the single layer potential on the boundary have been analyzed in [16]. They can be evaluated by a composite hp Gauss quadrature with geometrical grading towards the singularity [25, 17]. Higher derivatives have been analyzed by Schulz, Schwab and Wendland, e.g. in [26, 27], who compute the second normal derivative from the less singular tangential derivatives in a similar way. For the current problem, a simpler approach gives sufficient accuracy.

We investigate a general Dirichlet problem

$$
\begin{array}{rll}
-\Delta u=0 & \text { in } & \mathbb{R}^{d} \backslash \bar{\Omega} \\
u=f & \text { on } & \Gamma:=\partial \Omega
\end{array}
$$

with an appropriate decay condition. Using a single layer potential ansatz, the problem is equivalent to the integral equation $V \mu=f$, where

$$
u(x)=V \mu(x)=\int_{\Gamma} k(x, y) \mu(y) d s_{y}, \quad k(x, y)= \begin{cases}-\frac{1}{2 \pi} \log \|x-y\|, & d=2 \\ \frac{1}{4 \pi} \frac{1}{\|x-y\|}, & d=3 .\end{cases}
$$


As in Section 4, we solve the discretized Galerkin equations on the discretized boundary $\Gamma_{h}$,

$$
\left\langle V \mu_{h}, \xi\right\rangle_{\Gamma_{h}}=\langle g, \xi\rangle_{\Gamma_{h}} \quad \forall \xi \in S_{h, \Gamma}^{p}
$$

in the subspace $S_{h, \Gamma}^{p} \subset H^{-1 / 2}\left(\Gamma_{h}\right)$ of piecewise polynomials of degree $p$. To approximate the Hessian of $u$, we have to evaluate the Hadamard finite-part integral

$$
\nabla \nabla V \mu_{h}(x)=\text { p.f. } \int_{\Gamma_{h}} \nabla_{x} \nabla_{x} k(x, y) \mu_{h}(y) d s_{y} \quad\left(x \in \Gamma_{h}\right) .
$$

The hypersingular kernel $\nabla_{x} \nabla_{x} k(x, y)$ is the main challenge in evaluating the potential. We analytically compute the gradient $w=\nabla V \mu_{h}$ using the adjoint double layer potential. The second derivative $\nabla w$ is approximated by second-order accurate finite differences (FD):

$$
\begin{aligned}
& \frac{\partial w(x)}{\partial n}=\frac{4 w(x+\delta \cdot n)-3 w(x)-w(x+2 \delta \cdot n)}{2 \delta}+O\left(\delta^{2}\right) \\
& \frac{\partial w(x)}{\partial t}=\frac{w(x+\delta \cdot t)-w(x-\delta \cdot t)}{2 \delta}+O\left(\delta^{2}\right)
\end{aligned}
$$

In the computations, the step size $\delta$ is set to $10^{-4}$ for the normal component and to $10^{-5}$ for the tangential component when approximating second derivatives. For the presented numerical experiments the FD-approximation error is of magnitude $10^{-7}$ if no Galerkin-BEM approximation error were to occur. However, for very small step sizes the finite differences become numerically instable and the BEM-error is dominating. If $H=\left(H_{i j}\right)$ denotes the exact and $H_{h}=\left(H_{h, i j}\right)$ the approximated Hessian, we measure the error in a point $x$ as $\left(\sum_{i, j}\left(H_{h, i j}(x)-H_{i j}(x)\right)^{2}\right)^{1 / 2}$. The BEM-approximation error is measured in the energy norm $\left\|\mu-\mu_{h}\right\|_{V}^{2}:=\left\langle V\left(\mu-\mu_{h}\right), \mu-\mu_{h}\right\rangle_{\Gamma_{h}}$.

Example 1. Let $\Omega=\left[-\frac{1}{2}, \frac{1}{2}\right]^{2}$ be the domain and $u=\ln \|x\|$ the exact solution. Then the exact Hessian is $H(x)=\frac{1}{x^{2}}\left(\begin{array}{cc}1-2 x_{1}^{2} & -2 x_{1} x_{2} \\ -2 x_{1} x_{2} & 1-2 x_{2}^{2}\end{array}\right)$. Figure 1$]$ shows the pointwise error of the Hessian approximation in the point $x=\left(\frac{1}{2}, \frac{1}{3}\right)$ for $h$-versions of BEM with polynomial degree $p=0,1,2,3$, as well as for a $p$-version with $h=0.2$. Figure 2 displays the corresponding BEMerror $\left\|\mu-\mu_{h}\right\|_{V}$ between $\mu_{h}$ and an extrapolated density $\mu$. All versions show their characteristic rate of convergence, i.e. 1.5, 2.5, 3.5, 4.5 for the h-versions and exponential for the p-version until the error is about $10^{-8}$ at which point the quadrature errors for the outer integration in the semi-analytic evaluation of (25) dominate the BEM-error with analytic computation of the involved integrals.

Example 2. Let $\Omega=[-1,1]^{3}$ be the domain and $g$ corresponding to the exact solution $u(x)=\frac{1}{\|x\|}$ with Hessian $H(x)=\frac{3}{\|x\|^{5}}\left(\begin{array}{ccc}x_{1}^{2} & x_{1} x_{2} & x_{1} x_{3} \\ x_{1} x_{2} & x_{2}^{2} & x_{2} x_{3} \\ x_{1} x_{3} & x_{2} x_{3} & x_{3}^{2}\end{array}\right)-\frac{1}{\|x\|^{3}}$ I. Figure $[3$ displays the BEM-error $\left\|\mu-\mu_{h}\right\|_{V}$ for three h-versions $(p=0,1,2)$. Figure 4 shows the error in $x=\left(1, \frac{1}{3}, \frac{1}{3}\right)$. Again, at least for $p \geq 2$ we observe good convergence of the Hessian approximation. 


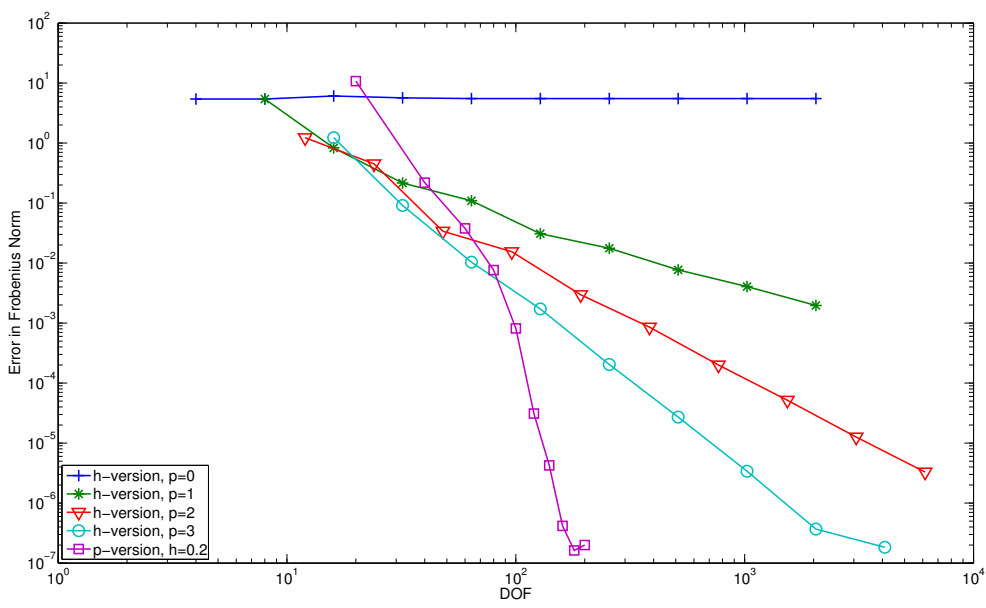

Figure 1: Error of the Hessian approximation for a point on $\Gamma_{h}$

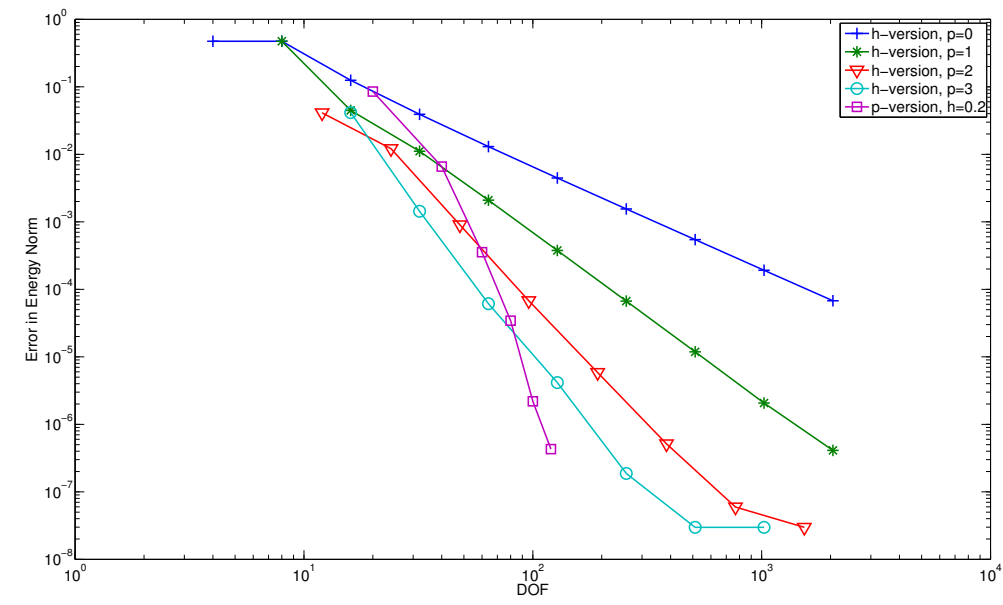

Figure 2: BEM-Error $\left\|\mu-\mu_{h}\right\|_{V}$ in the energy norm for the $2 \mathrm{~d}$ case

Similar qualitative results hold for the case of a sphere. However, in this case the error for the domain approximation quickly dominates the numerical error of differentiation [20].

To control the error of the domain approximation, it is well known [28] that the finite elements to approximate the surface should be one order higher than the finite elements to approximate the solution of the integral equation with the single layer potential. As the accurate computation of the Hessian requires at least quadratic elements, third order elements should be used for $\varphi$. The corresponding software development will require further research. 


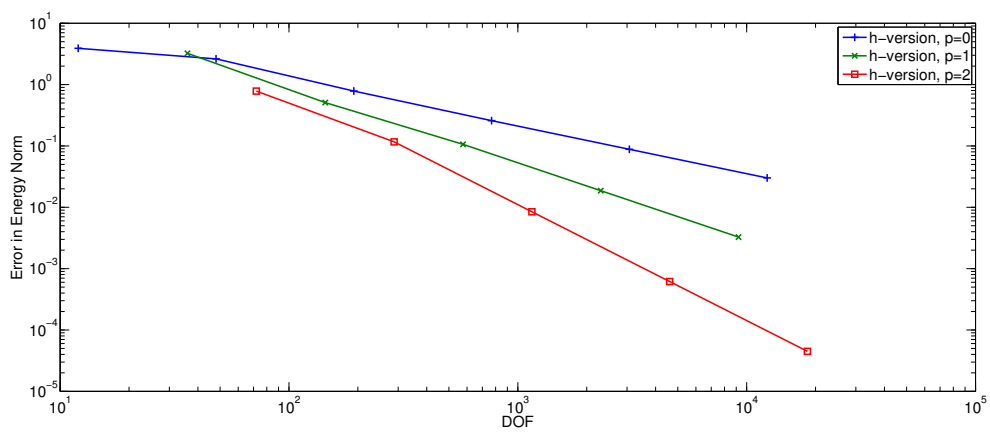

Figure 3: BEM-Error $\left\|\mu-\mu_{h}\right\|_{V}$ in the energy norm for the $3 \mathrm{~d}$ case on the cube

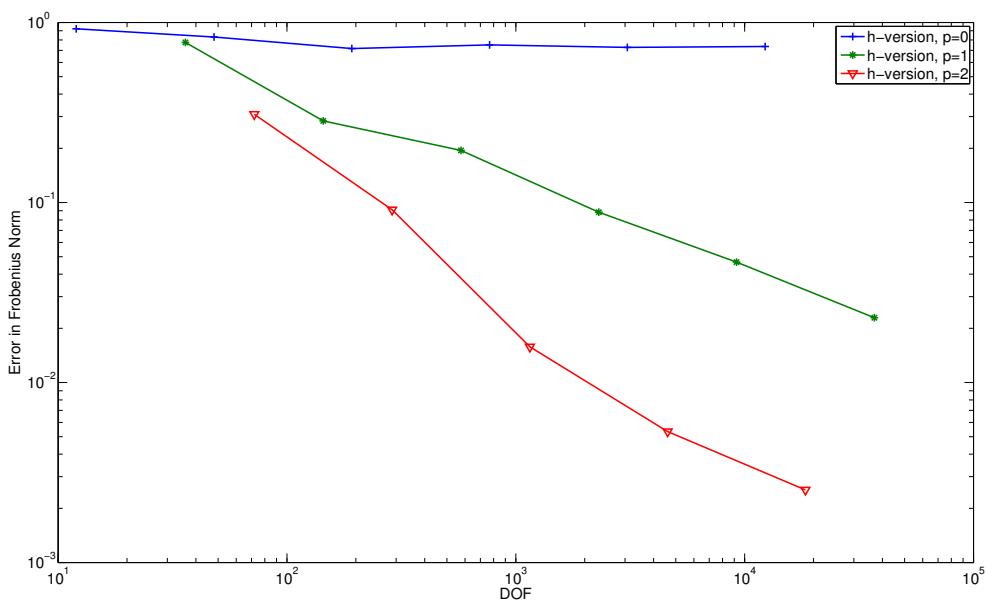

Figure 4: Error of the Hessian approximation 3d case, $h$-versions for a point on $\Gamma$

\subsection{Molodensky problem}

In this section we perform a first study of the numerical solution of the fully nonlinear Molodensky problem. To assess the accuracy and convergence properties of the proposed algorithm, we study it in a simple, but algorithmically nontrivial model problem with an explicit exact solution.

The data $W, G$ are those of a spherical earth of radius 1.1 with the Newton gravitational potential: For $x \in S^{2}$, the surface is described by $\varphi(x)=1.1 x$, the gravitational potential is $W(x)=\frac{1}{1.1}$, and $G(x)=-\frac{x}{1.1^{2}}$. We choose the unit sphere $S^{2}, \varphi_{0}(x)=x$, as initial approximation. Therefore, $W_{0}(x)=1, G_{0}(x)=-x$ and $h_{0}=\frac{x}{2}\left(x \in S^{2}\right)$.

Since our model problem is rotationally invariant and the algorithm preserves this symmetry, we expect a sequence of computed surfaces which are slightly perturbed spheres converging to a sphere of radius 1.1. The perturbation should be due to discretization and rounding errors. The 
mean $L^{2}$ error in $\varphi$ is thus equivalent to

$$
\left\|\varphi_{m}-\varphi\right\|_{L^{2}} \asymp \frac{1}{\# \text { nodes }}\left[\sum_{i=1}^{\# \text { nodes }} \operatorname{dist}\left(\varphi_{m}\left(x_{i}\right), \varphi\left(S^{2}\right)\right)^{2}\right]^{1 / 2} .
$$

We have performed several numerical experiments with different parameters $\theta_{0}, \kappa$. Firstly, if the amount of data smoothing is too small, the algorithm is unstable as expected (and observed) in the case without smoothing operator. Secondly, if the amount of data smoothing is too large, then essential information on the right hand side in the linearized Molodensky problem is lost in the first steps, and in combination with the numerical errors convergence is lost. Also if the amount of smoothing does not decay sufficiently fast, the right hand side in the linearized Molodensky problem is close to machine precision, leading to numerical artefacts. Figure 5 presents some reasonable choices of parameters $\theta_{0}, \kappa$.

Figure 5 shows that the propagation of the discretization error cannot be eliminated. However, increasing the amount of smoothing per iteration for a fixed mesh delays the point at which the propagated discretization error becomes dominating. Decreasing the mesh size, leads to a more even error reduction per iteration step. However, the error reduction per iteration step also decreases. The point at which discretisation errors become dominant seems difficult to predict.

Figure 6 shows the average pointwise error

$$
\left|u_{N}(x)-u(x)\right|_{a v}=\frac{1}{M}\left(\sum_{i=1}^{M}\left|u_{N}\left(x_{i}^{e x t}\right)-u\left(x_{i}^{e x t}\right)\right|^{2}\right)^{1 / 2}
$$

computed in a set of $M=10242$ exterior points for the linearized Molodensky problem with smoother $\left(\theta_{0}=2.6, \kappa=6\right)$ for the first three Nash-Hörmander iteration steps. Here $u(x)$ is obtained by extrapolation. All three curves show similar convergence rates for DOF $\rightarrow \infty$, see Table 1.

Figure 7 displays the $L^{2}$ error in $\varphi$ versus the number of restarts for the restarted algorithm. The restart is done after each iteration step. We observe the same structural behaviour as for the other two experiments. Therefore, from the third restart of the algorithm onwards the discretization error propagation becomes dominating. However, refining the mesh, from $N=2$ to $N=3$, slightly reduces the error after the second and third restart, before increasing after the third restart.

Figure 8 displays the $L^{2}$ error in the gravity vector,

$$
\left\|G_{m}-G\right\|_{L^{2}} \asymp \frac{1}{\# \text { nodes }}\left[\sum_{i=1}^{\# \text { nodes }}\left(G_{m}\left(x_{i}\right)-G\left(x_{i}\right)\right)^{2}\right]^{1 / 2},
$$

in the algorithm with restart. The errors slowly decreases for the first five iteration steps, but from this step onwards the method provides uncontrollable surface updates (peaks and undesirable deformations occur) afterwards.

To sum up, we observe convergence of the algorithm in $\varphi$ until numerical errors due to discretization and domain approximation accumulate. A higher-order discretization of the surface seems to be necessary to obtain stable convergence and an improved approximation of $G$ after 


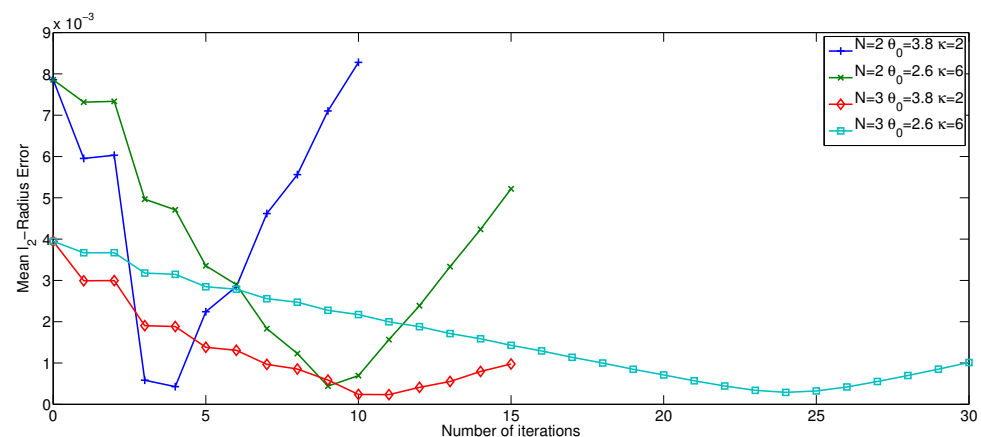

Figure 5: $\left\|\varphi_{m}-\varphi\right\|_{L^{2}}$ for the algorithm without restart

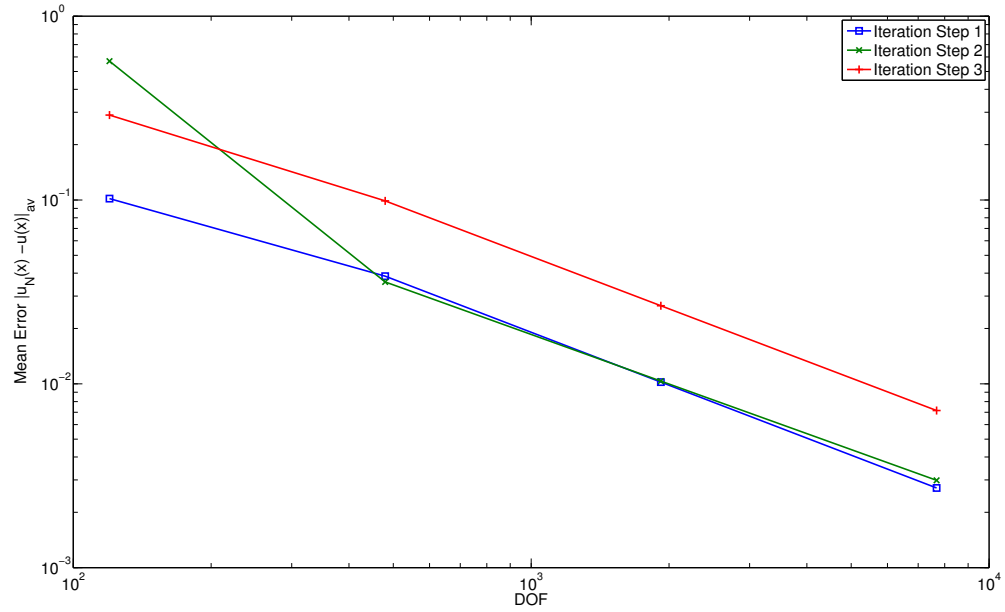

Figure 6: Pointwise error $\left|u_{N}(x)-u(x)\right|_{a v}$ computed in a set of 10242 exterior points for the linearized Molodensky problem 


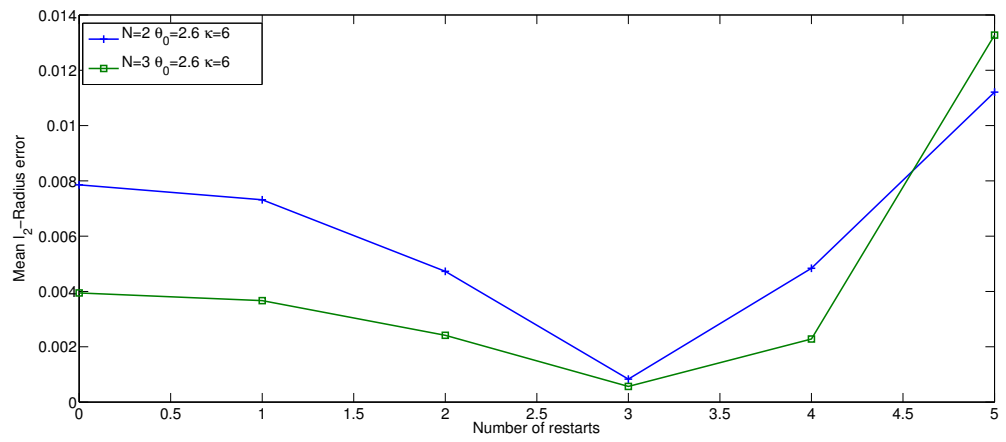

Figure 7: $\left\|\varphi_{m}-\varphi\right\|_{L^{2}}$ for the algorithm with restart

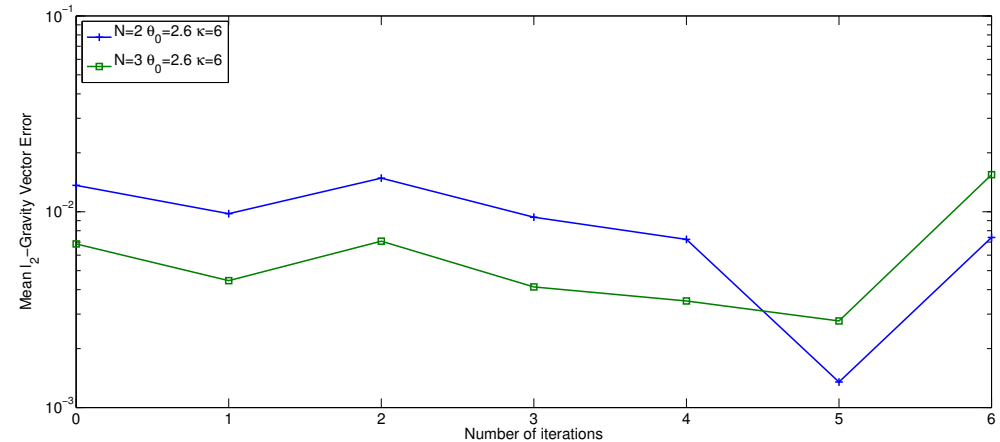

Figure 8: $\left\|G_{m}-G\right\|_{L^{2}}$ for the algorithm with restart

\begin{tabular}{|r|r|c|l|}
\hline Iter & DOF & $\left|u_{N}(x)-u(x)\right|_{a v}$ & EOC \\
\hline 0 & 120 & 0.10170 & \\
& 480 & 0.03850 & 0.70 \\
& 1920 & 0.01022 & 0.96 \\
& 7680 & 0.00271 & 0.96 \\
\hline 1 & 120 & 0.56875 & \\
& 480 & 0.03582 & 1.99 \\
& 1920 & 0.01034 & 0.90 \\
& 7680 & 0.00299 & 0.90 \\
\hline 2 & 120 & 0.28961 & \\
& 480 & 0.09885 & 0.77 \\
& 1920 & 0.02660 & 0.95 \\
& 7680 & 0.00716 & 0.95 \\
\hline
\end{tabular}

Table 1: Pointwise errors and experimental orders of convergence for the linearized Molodensky problem 
a larger number of steps. For the restarted algorithm, a better understanding of the optimal smoothing parameters must be achieved.

Figure 9 displays the sequence of obtained surfaces. The marked point is always the north pole of the sphere, i.e. $x=y=0$ and only $z$ varies. Interestingly, for each experiment the surface update is almost constant over the mesh points, leading to a sequence of almost spheres.

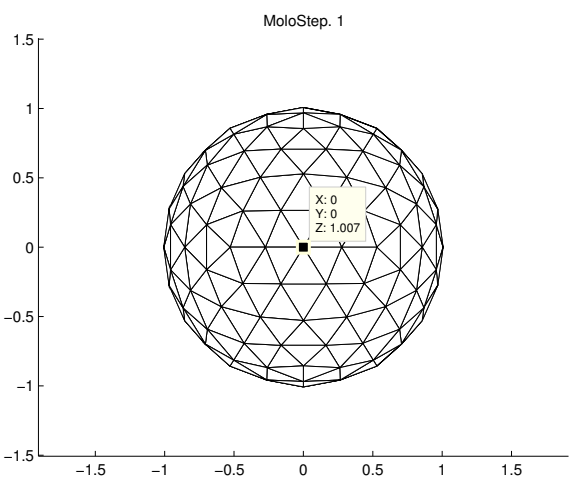

(a) 320 triangles, $z=1.007$

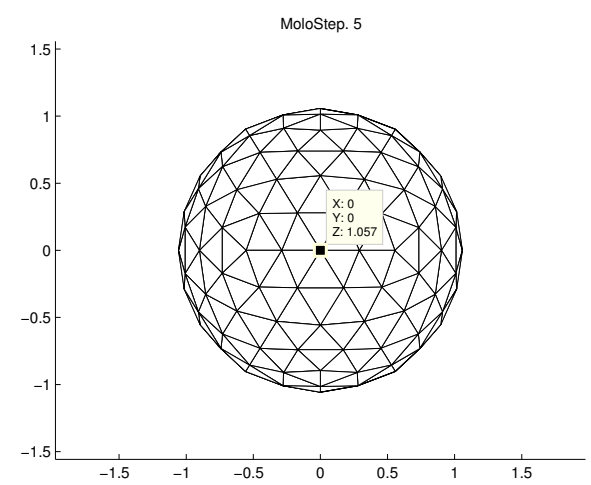

(c) 320 triangles, $z=1.057$

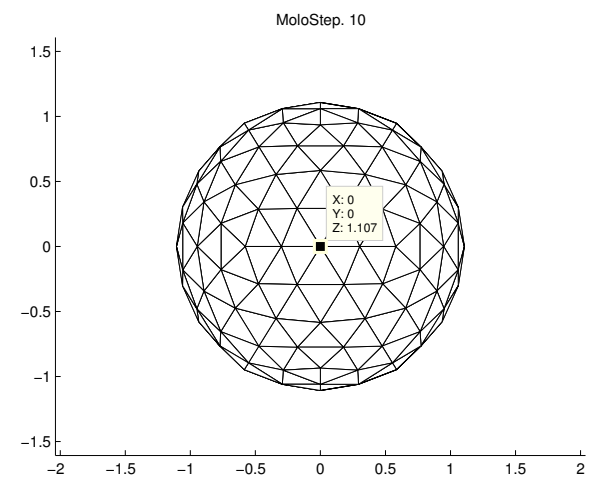

(e) 320 triangles, $z=1.107$

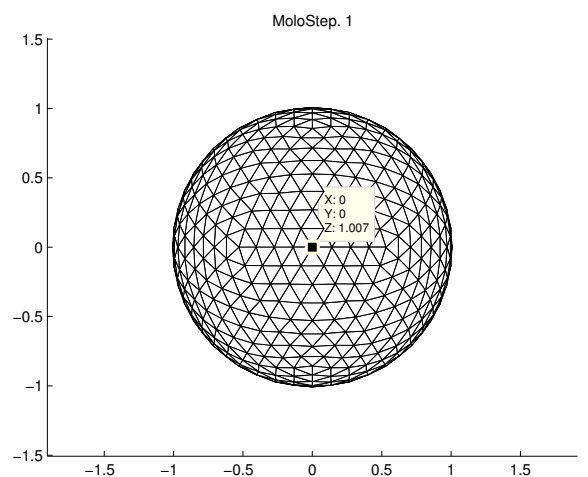

(b) 1280 triangles, $z=1.007$

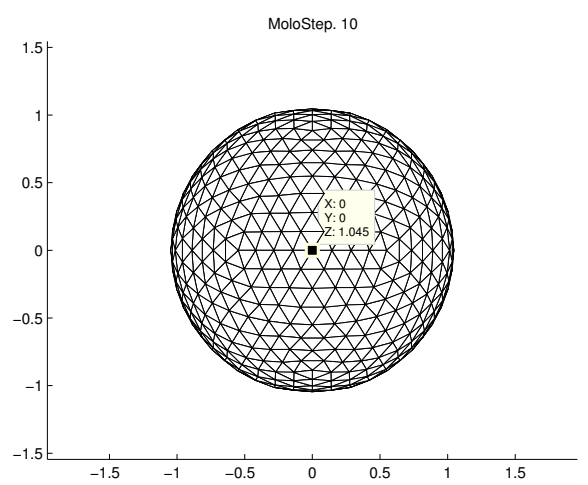

(d) 1280 triangles, $z=1.045$

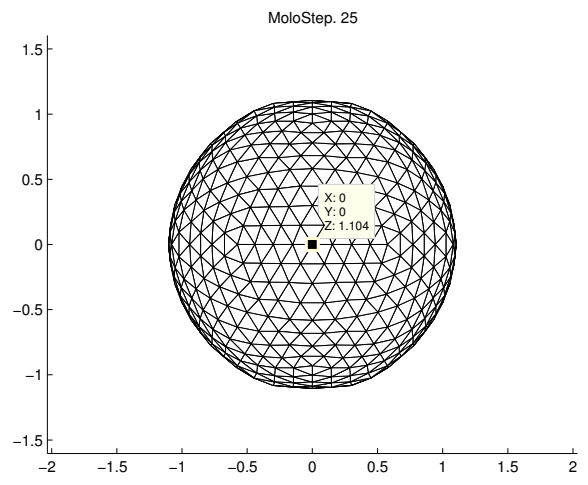

(f) 1280 triangles, $z=1.104$

Figure 9: Icosahedron refinements for $\theta_{0}=2.6, \kappa=6$ 


\section{Appendix}

We are going to use the following definition of the Hölder-spaces, but will rarely use them for integer exponent $a$ :

Definition 1 (Definition A.3 [5]). Let $k \in \mathbb{N}_{0}, k<a \leq k+1$ and $B \subseteq \mathbb{R}^{n}$ compact, convex such that $\stackrel{\circ}{B} \neq \emptyset$.

Define

$$
\begin{aligned}
\mathscr{H}^{a}(B):=\left\{u \in C^{k}(B):\|u\|_{0}\right. & =\sup _{x \in B}|u(x)|<\infty \text { and } \\
|u|_{a} & \left.:=\sum_{|\alpha|=k} \sup _{x \neq y \in B} \frac{\left|\partial^{\alpha} u(x)-\partial^{\alpha} u(y)\right|}{|x-y|^{a-k}}<\infty\right\} .
\end{aligned}
$$

We also set $\mathscr{H}^{0}(B):=C(B)$. Then $\mathscr{H}^{a}:=\mathscr{H}^{a}(B)$ with the norm $\|\cdot\|_{a}:=\|\cdot\|_{0}+|\cdot|_{a}$ is a Banach space.

For a compact manifold, one defines $\mathscr{H}^{a}$ by covering it with a finite number of neighborhoods homeomorphic to subsets of $\mathbb{R}^{n}$.

Basic interpolation estimates will be used frequently:

$$
\|v\|_{\sigma a+(1-\sigma) b} \leq C\|v\|_{a}^{\sigma}\|v\|_{b}^{1-\sigma} \quad\left(\sigma \in(0,1), v \in \mathscr{H}^{\max \{a, b\}}\right) .
$$

Similarly, if $(a, b) \in \mathbb{R}_{+}^{2}$ belongs to the convex hull of $\left(a_{1}, b_{1}\right), \ldots,\left(a_{J}, b_{J}\right) \in \mathbb{R}_{+}^{2}$, then

$$
\|v\|_{a}\|w\|_{b} \leq C \sum_{j=1}^{J}\|v\|_{a_{j}}\|w\|_{b_{j}} \quad\left(v \in \mathscr{H}^{\max \left\{a_{j}\right\}}, w \in \mathscr{H}^{\max \left\{b_{j}\right\}}\right) .
$$

\subsection{Proof of Theorem 2}

For ease of presentation, we set $u=\left(u^{(1)}, u^{(2)}\right):=(W, \varphi): S^{2} \rightarrow \mathbb{R} \times \mathbb{R}^{3}, f:=(G, W): S^{2} \rightarrow$ $\mathbb{R}^{3} \times \mathbb{R}$. The map from $(W, \varphi)$ to the corresponding $G$ is denoted by $\Gamma$. The Molodensky problem assumes the form $\Phi(u):=\left(\Gamma(u), u^{(1)}\right)=f$.

In this notation, the Nash-Hörmander iteration reads as

$$
u_{m+1}=u_{m}+\Delta_{m} \dot{u}_{m}, \quad \dot{u}_{m}=\Psi\left(v_{m}\right) g_{m}, \quad v_{m}=S_{\theta_{m}} u_{m},
$$

$\Psi$ being the inverse operator to the linearization of $\Phi$ around $v_{m}$. To show that the Algorithm 1 is a reformulation of the one in [5], we show that the equations (15) and (16) are equivalent to usual definition

$$
\Delta_{0} g_{0}=S_{\theta_{0}} f, \quad g_{m}=\Delta_{m}^{-1}\left(\left(S_{\theta_{m}}-S_{\theta_{m-1}}\right)\left(f-E_{m-1}\right)-\Delta_{m-1} S_{\theta_{m}} e_{m-1}\right) \quad(m>0) .
$$

Here, the errors $e_{m}=e_{m}^{\prime}+e_{m}^{\prime \prime}$ are defined as the sum of a smoothing error

$$
e_{m}^{\prime}=\left(\Phi^{\prime}\left(u_{m}\right)-\Phi^{\prime}\left(v_{m}\right)\right) \dot{u}_{m}
$$


and a linearization error

$$
e_{m}^{\prime \prime}=\Delta_{m}^{-1}\left(\Phi\left(u_{m}+\Delta_{m} \dot{u}_{m}\right)-\Phi\left(u_{m}\right)-\Delta_{m} \Phi^{\prime}\left(u_{m}\right) \dot{u}_{m}\right)
$$

The total error is $E_{m}=e_{0}+\cdots+e_{m-1}\left(e_{0}=E_{0}=0\right)$.

In our case, $\Phi^{\prime}(u) \dot{u}=\left(\Gamma^{\prime}(u) \dot{u}, \dot{u}^{(1)}\right)$, so that with $\dot{G}_{m}=\Gamma^{\prime}\left(W_{m}, \varphi_{m}\right)\left(\dot{W}_{m}, \dot{\varphi}_{m}\right)$

$$
\begin{aligned}
e_{m}^{\prime} & =\Phi^{\prime}\left(W_{m}, \varphi_{m}\right)\left(\dot{W}_{m}, \dot{\varphi}_{m}\right)-\Phi^{\prime}\left(\widetilde{W}_{m}, \widetilde{\varphi}_{m}\right)\left(\dot{W}_{m}, \dot{\varphi}_{m}\right) \\
& =\left(\Gamma^{\prime}\left(W_{m}, \varphi_{m}\right)\left(\dot{W}_{m}, \dot{\varphi}_{m}\right), \dot{W}_{m}\right)-\left(\Gamma^{\prime}\left(\widetilde{W}_{m}, \widetilde{\varphi}_{m}\right)\left(\dot{W}_{m}, \dot{\varphi}_{m}\right), \dot{W}_{m}\right) \\
& =\left(\dot{G}_{m}-g_{m}^{(1)}, 0\right)
\end{aligned}
$$

Similarly, for the linearization error we have

$$
\Delta_{m} e_{m}^{\prime \prime}=\Phi\left(W_{m}+\Delta_{m} \dot{W}_{m}, \varphi_{m}+\Delta_{m} \dot{\varphi}_{m}\right)-\Phi\left(W_{m}, \varphi_{m}\right)-\Delta_{m} \Phi^{\prime}\left(W_{m}, \varphi_{m}\right)\left(\dot{W}_{m}, \dot{\varphi}_{m}\right),
$$

with second component $W_{m}+\Delta_{m} \dot{W}_{m}-W_{m}-\Delta_{m} \dot{W}_{m}=0$. The first component, by definition, is $G_{m+1}-G_{m}-\Delta_{m} \dot{G}_{m}$.

As a consequence, the second components of the errors $e_{m}$ and $E_{m}$ vanish. Equation (29) yields

$$
\Delta_{0} g_{0}^{(2)}=S_{\theta_{0}}\left(W-W_{0}\right), \quad \Delta_{m} g_{m}^{(2)}=S_{\theta_{m}}\left(W-W_{0}\right)-S_{\theta_{m-1}}\left(W-W_{0}\right) \quad(m>0) .
$$

Concerning the first component of $g_{m}$, we use $\Delta_{0} g_{0}^{(1)}=S_{\theta_{0}}\left(G-G_{0}\right)$ and our above computation:

$$
\begin{aligned}
\Delta_{1} g_{1}^{(1)} & =\left(S_{\theta_{1}}-S_{\theta_{0}}\right)\left(G-G_{0}\right)-\Delta_{0} S_{\theta_{1}}\left(\dot{G}_{0}-g_{0}^{(1)}\right)-S_{\theta_{1}}\left(G_{1}-G_{0}-\Delta_{0} \dot{G}_{0}\right) \\
& =S_{\theta_{1}}\left(G-G_{1}+\Delta_{0} g_{0}^{(1)}\right)-S_{\theta_{0}}\left(G-G_{0}\right) .
\end{aligned}
$$

Similarly we obtain a recursion formula for $m>1$ :

$$
\Delta_{m} g_{m}^{(1)}=S_{\theta_{m}}\left(G-G_{m}+\sum_{j=0}^{m-1} \Delta_{j} g_{j}^{(1)}\right)-S_{\theta_{m-1}}\left(G-G_{m-1}+\sum_{j=0}^{m-2} \Delta_{j} g_{j}^{(1)}\right)
$$

Equations (32) and (33) show the equivalence of Algorithm 1 to the formulation in [5].

of Theorem 2. By the above reduction, it is sufficient to analyze Hörmander's iterative method and derive an a priori estimate for the error after $m$ steps. Our proof relies on certain estimates in Hörmander's qualitative analysis. Recall that the smoothing operator is generated by $(-1)^{k} \Delta^{k}$.

The proof is given in several steps. We are going to rely on a number of auxiliary results.

Given a sufficently large, fixed $a_{\Phi}$, we recall the following continuity estimates for $\Phi^{\prime \prime}$ and the inverse of the linearization $\Psi$ from ([5], equations $(2.1 .5 / 6))$ :

1.) For all $\epsilon>0,0 \leq a \leq a_{\Phi}$ and $u, v, w \in C^{\infty}$ with $\|u\|_{2+\epsilon}<C$ we have:

$$
\left\|\Phi^{\prime \prime}(u ; v, w)\right\|_{a+2 \epsilon} \lesssim\|v\|_{a+2+3 \epsilon}\|w\|_{0}+\|v\|_{0}\|w\|_{a+2+3 \epsilon}+\|v\|_{0}\|w\|_{0}\|u\|_{a+3+2 \epsilon}
$$


2.) For all $\epsilon>0,0 \leq a \leq a_{\Phi}$ and $v, g \in C^{\infty}$ with $\|v\|_{2+\epsilon}<C$

$$
\|\Psi(v) g\|_{a+\epsilon} \lesssim\|g\|_{a+\epsilon}+\|g\|_{\epsilon}\|v\|_{a+2+\epsilon}
$$

The first lemma translates a bound for the first $m$ increments $\dot{u}_{j}$ into properties of $U_{m}=$ $\sum_{j=0}^{m} \triangle_{j} \dot{u}_{j}$

Lemma 1. Let $\epsilon>0, \alpha+\epsilon \notin \mathbb{N},-\epsilon \leq \alpha_{-} \leq \alpha \leq \alpha_{+}$. Assume that $2 k>\alpha+\epsilon-a$ and that for some $\delta>0, m \geq 0$

$$
\left\|\dot{u}_{j}\right\|_{a+\epsilon} \leq \delta \theta_{j}^{a-\alpha-1} \quad \forall 0 \leq j \leq m \forall a \in\left[\alpha_{-}, \alpha_{+}\right] .
$$

Then $U_{m}=\sum_{j=0}^{m} \triangle_{j} \dot{u}_{j} \in \mathscr{H}^{\alpha+\epsilon}$ satisfies

$$
\begin{aligned}
\left\|U_{m}\right\|_{a} & \leq C_{1} \delta \quad \forall a \leq \alpha+\epsilon, \\
\left\|U_{m}-S_{\theta_{m+1}} U_{m}\right\|_{a} & \leq C_{2} \delta \theta_{m+1}^{a-\alpha-\epsilon} \quad \forall 0 \leq a \leq \alpha_{+}+\epsilon, \\
\left\|S_{\theta_{m+1}} U_{m}\right\|_{a} & \leq C_{3} \delta \theta_{m+1}^{(a-\alpha-\epsilon)_{+}} \quad \forall 0 \leq a \leq a_{0}
\end{aligned}
$$

for fixed $a_{0}$.

We refer to [5, Lemma 2.2.1]) for the proof.

The lemma implies that under the given assumptions all iterates $u_{m}$ will remain in a neighborhood of $u_{0}$. We may therefore localize and appeal to estimates valid near $u_{0}$ and justify the linearization of the problem. A quantitative formulation of the localization is as follows:

Corollary 1. Let $\widetilde{\epsilon}>0, \mu \leq \alpha+\epsilon$, and $a, \delta$ as above. Define

$$
\begin{aligned}
& V_{s}:=\left\{u \in C^{\infty}:\left\|u-u_{0}\right\|_{\mu} \leq s\right\}, \\
& \widetilde{V}_{s}:=\left\{u \in C^{\infty}:\|u\|_{\mu} \leq s\right\} .
\end{aligned}
$$

Then there exist $C, C^{\prime}>0$ (which depend only on $C_{1}$ and the constants in Theorem $3(i)$, (iii')) such that for all $\theta \geq C\left(\frac{\tilde{\epsilon}}{\left\|u_{0}\right\|_{a}}\right)^{\frac{1}{a-\mu}}$ and all $\delta<C^{\prime} \tilde{\epsilon}$ :

a) $S_{\theta} u_{0} \in V_{\widetilde{\epsilon} / 2}$

b) If $a=\mu \leq \alpha+\epsilon$, then $U_{k}, S_{\theta} U_{k} \in \widetilde{V}_{\widetilde{\epsilon} / 2}, u_{k+1}=u_{0}+U_{k} \in V_{\widetilde{\epsilon}}$ and $S_{\theta_{k+1}} u_{k+1} \in V_{\widetilde{\epsilon}}$.

We note some related estimates for the smoothed iterates $v_{m}=S_{\theta_{m}} u_{m}$, which in particular hold for $b=\alpha+\epsilon$ :

Corollary 2. Under the above assumptions there holds:

$$
\begin{aligned}
\left\|u_{j}-v_{j}\right\|_{c} \leq C\left(\left\|u_{0}\right\|_{b} \theta_{j}^{c-b}+\delta \theta_{j}^{c-\alpha-\epsilon}\right) & \forall c \leq \alpha+\epsilon, 0 \leq b-c<2 k \\
\left\|v_{j}\right\|_{c} \leq C\left(\left\|u_{0}\right\|_{b} \theta_{j}^{c-b}+\delta \theta_{j}^{(c-\alpha-\epsilon)_{+}}\right) & \forall c \leq c_{0}, b \leq c
\end{aligned}
$$

Proof. Indeed, using Property (iii') of $S_{\theta}$ and Lemma 1 .

$$
\begin{aligned}
\left\|u_{j}-v_{j}\right\|_{c} & \leq\left\|u_{0}-S_{\theta_{j}} u_{0}\right\|_{c}+\left\|U_{j-1}-S_{\theta_{j}} U_{j-1}\right\|_{c} \\
& \leq C\left\|u_{0}\right\|_{b} \theta_{j}^{c-b}+C \delta \theta_{j}^{c-\alpha-\epsilon} .
\end{aligned}
$$

Similarly, $\left\|v_{j}\right\|_{c} \leq\left\|S_{\theta_{j}} u_{0}\right\|_{c}+\left\|S_{\theta_{j}} U_{j-1}\right\|_{c} \leq C \theta_{j}^{c-b}\left\|u_{0}\right\|_{b}+C \delta \theta_{j}^{(c-\alpha-\epsilon)_{+}}$ 
The a priori estimate of Theorem 2 will be shown by induction in $m$.

As hypothesis we assume (36), that $\|f\|_{\alpha+\epsilon}$ is small and that the assumptions of Lemma 1 are verified for a suitable $\delta$. We are going to deduce the corresponding assertions with $m$ replaced by $m+1$.

Together with the induction hypothesis, Lemma 1 and the above corollaries provide bounds of the Hölder norms of $u_{j}, v_{j}$ and $u_{j}-v_{j}$ for $0 \leq j \leq m$. To estimate $\dot{u}_{m+1}$ in terms of these data, note that by definition of $\dot{u}_{m+1}$ and $g_{m+1}$,

$$
\begin{aligned}
\left\|\dot{u}_{m+1}\right\|_{a+\epsilon} & \leq C\left(\left\|g_{m+1}\right\|_{a+\epsilon}+\left\|g_{m+1}\right\|_{\epsilon} \theta_{m+1}^{(2+a-\alpha)_{+}}\right) \\
g_{m+1} & =\widetilde{S}_{m}\left(f-E_{m}\right)-\frac{\triangle_{m}}{\triangle_{m+1}} S_{\theta_{m+1}} e_{m}
\end{aligned}
$$

where $E_{m}=\sum_{j=0}^{m-1} \triangle_{j} e_{j}$ and $\widetilde{S}_{m}=\frac{S_{\theta_{m+1}}-S_{\theta_{m}}}{\theta_{m+1}-\theta_{m}}$. Writing $\widetilde{S}_{m}$ as an average of $\frac{d}{d \theta} S_{\theta}, \widetilde{S}_{m} f$ can be bounded using Property (iv) of the smoothing operator,

$$
\left\|\tilde{S}_{m} f\right\|_{b} \leq C \theta_{m}^{b-c-1}\|f\|_{c},
$$

for any $b, c$. Properties (ii) and (iv) of the smoothing operator give similar estimates for the other terms:

$$
\begin{aligned}
\left\|S_{\theta_{m+1}} e_{m}\right\|_{b} & \leq C \theta_{m}^{\left(b-c^{\prime}\right)+}\left\|e_{m}\right\|_{c^{\prime}} \\
\left\|\widetilde{S}_{m} E_{m}\right\|_{b} & \leq \theta_{m}^{b-c^{\prime \prime}-1} \sum_{j=0}^{m-1} \triangle_{j}\left\|e_{j}\right\|_{c^{\prime \prime}}
\end{aligned}
$$

Hence

$$
\begin{gathered}
\left\|g_{m+1}\right\|_{a+\varepsilon} \lesssim \theta_{m}^{a+\varepsilon-c-1}\|f\|_{c}+\theta_{m}^{a+\varepsilon-c^{\prime \prime}-1} \sum_{j=0}^{m-1} \triangle_{j}\left\|e_{j}\right\|_{c^{\prime \prime}}+\theta_{m}^{\left(a+\varepsilon-c^{\prime}\right)_{+}}\left\|e_{m}\right\|_{c^{\prime}} \\
\theta_{m+1}^{(2+a-\alpha)_{+}}\left\|g_{m+1}\right\|_{\varepsilon} \lesssim \theta_{m}^{\varepsilon-C-1+(2+a-\alpha)_{+}}\|f\|_{C}+\theta_{m}^{\varepsilon-C^{\prime \prime}-1+(2+a-\alpha)_{+}} \sum_{j=0}^{m-1} \triangle_{j}\left\|e_{j}\right\|_{C^{\prime \prime}} \\
+\theta_{m}^{\left(\varepsilon-C^{\prime}\right)_{+}+(2+a-\alpha)_{+}}\left\|e_{m}\right\|_{C^{\prime}}
\end{gathered}
$$

giving a bound on $\dot{u}_{m+1}$. If we choose $c=\alpha+\varepsilon, C=\alpha-a+\varepsilon+(2+a-\alpha)_{+} \leq \alpha+\varepsilon$, the terms involving $f$ are dominated by $\theta_{m+1}^{E}\|f\|_{\alpha+\epsilon}$

To estimate $e_{j}$, we will consider the smoothing error $e_{j}^{\prime}$ and the linearization error $e_{j}^{\prime \prime}$ separately. At the end of this section we use (34) and (35) to bound $e_{j}^{\prime}$ resp. $e_{j}^{\prime \prime}$ in terms of the Hölder norms of $u_{j}, v_{j}$ and $u_{j}-v_{j}$ for $0 \leq j \leq m$, which are controlled by the induction hypothesis. A computation eventually results in

$$
\left\|\dot{u}_{m+1}\right\|_{a+\epsilon} \leq C \theta_{m+1}^{E}\left(A\|f\|_{\alpha+\epsilon}+\delta \sigma\left(1+\delta+\delta^{2}\right)\right)
$$


for small $\sigma \in(0,1)$ whenever $\theta_{0}=\theta_{0}\left(\sigma, u_{0}, \alpha, S_{\theta}\right)$ is sufficiently large. $A$ only depends on the constants in (35) and Property (iv) of the smoothing operator. Both $\theta_{0}$ and $A$ are, in principle, explicit. We choose $\sigma=\frac{1}{2 C} \frac{1}{1+\delta+\delta^{2}}$.

Then for all $f$ in the ball $\left\{u:\|u\|_{\alpha+\epsilon} \leq \frac{\delta}{2 A C}\right\}$ we have

$$
\left\|\dot{u}_{m+1}\right\|_{a+\epsilon} \leq \delta \theta_{m+1}^{E}
$$

On the other hand, in the first step the solution to the linearized problem $\dot{u}_{0}=\triangle_{0}^{-1} \Psi\left(S_{\theta_{0}} u_{0}\right) S_{\theta_{0}} f$ is easily estimated using (35) and the smoothing properties

$$
\begin{aligned}
\triangle_{0}\left\|\dot{u}_{0}\right\|_{a+\epsilon} & \leq C^{\prime}\left(\left\|S_{\theta_{0}} f\right\|_{a+\epsilon}+\left\|S_{\theta_{0}} f\right\|_{\epsilon}\left\|S_{\theta_{0}} u_{0}\right\|_{a+2+\epsilon}\right) \\
& \leq C^{\prime \prime} \theta_{0}\left(1+\theta_{0}^{-a}\left\|u_{0}\right\|_{2+\epsilon+a}\right)\|f\|_{\alpha+\epsilon} \theta_{0}^{E} .
\end{aligned}
$$

We now denote by $\mathfrak{C}$ the maximum of $C^{\prime \prime} \triangle_{0}^{-1} \theta_{0}\left(1+\theta_{0}^{-a}\left\|u_{0}\right\|_{2+\epsilon+a}\right)$ and the previous constant $2 A C$ and choose $\delta=\mathfrak{C}\|f\|_{\alpha+\epsilon}$. Since $\|f\|_{\alpha+\epsilon}$ was sufficiently small by hypothesis, so is $\delta$, and (47) is fulfilled. By induction, we deduce

$$
\left\|\dot{u}_{m+1}\right\|_{a+\epsilon} \leq \mathfrak{C}\|f\|_{\alpha+\epsilon} \theta_{m+1}^{E} \quad \forall k \geq 0 .
$$

If $u$ denotes the exact solution, we obtain

$$
\begin{aligned}
\left\|u-u_{m}\right\|_{a+\epsilon} & \leq \sum_{j=m+1}^{\infty} \triangle_{j}\left\|\dot{u}_{j}\right\|_{a+\epsilon} \leq \mathfrak{C}\|f\|_{\alpha+\epsilon} \sum_{j=m+1}^{\infty} \triangle_{j} \theta_{j}^{E} \\
& \leq C_{\tau} \mathfrak{C}\|f\|_{\alpha+\epsilon} \theta_{m}^{E+1+\tau}
\end{aligned}
$$

for any $\tau>0$ small such that $E+1+\tau<0$. As $u$ was the exact solution, this yields the assertion of Theorem 2 ,

Note that

$$
\Phi\left(u_{m+1}\right)-\Phi(u)=\Phi\left(u_{m+1}\right)-\Phi\left(u_{0}\right)-f=\left(S_{\theta_{m}} f-f\right)+\triangle_{m} e_{m}+\left(E_{m}-S_{\theta_{m}} E_{m}\right) .
$$

We have shown that the right hand side converges to 0 in $\mathcal{H}^{a+\epsilon}$, so that also $\Phi\left(u_{m}\right)$ converges to $\Phi(u)$.

To complete the proof, it remains to estimate $e_{m}$ and to translate the result into a bound on $\dot{u}_{m+1}$. For the $j$-th smoothing error

$$
e_{j}^{\prime}=\left(\Phi^{\prime}\left(u_{j}\right)-\Phi^{\prime}\left(v_{j}\right)\right) \dot{u}_{j}=\int_{0}^{1} \Phi^{\prime \prime}\left(v_{j}+t\left(u_{j}-v_{j}\right) ; u_{j}-v_{j}, \dot{u}_{j}\right) d t
$$

equation (34) implies

$$
\begin{aligned}
\left\|e_{j}^{\prime}\right\|_{2 \epsilon+a} \lesssim\left\|u_{j}-v_{j}\right\|_{2+3 \epsilon+a}\left\|\dot{u}_{j}\right\|_{0}+\left\|u_{j}-v_{j}\right\|_{0}\left\|\dot{u}_{j}\right\|_{2+3 \epsilon+a} \\
+2\left\|u_{j}-v_{j}\right\|_{0}\left\|\dot{u}_{j}\right\|_{0}\left(\left\|u_{j}\right\|_{3+2 \epsilon+a}+\left\|v_{j}\right\|_{3+2 \epsilon+a}\right) .
\end{aligned}
$$


Using Lemma 1. $\left\|u_{j}\right\|_{b} \leq\left\|u_{0}\right\|_{b}+C \delta$, and the estimates for $v_{j}$ and $u_{j}-v_{j}$ from (40), (41), we obtain

$$
\begin{gathered}
\left\|e_{j}^{\prime}\right\|_{2 \epsilon+a} \lesssim\left(\left\|u_{0}\right\|_{b} \theta_{j}^{2+3 \epsilon+a-b}+\delta \theta_{j}^{2+2 \epsilon+a-\alpha}\right) \delta \theta_{j}^{-\alpha-1}+\left(\left\|u_{0}\right\|_{c_{1}} \theta_{j}^{-c_{1}}+\delta \theta_{j}^{-\alpha-\epsilon}\right) \delta \theta_{j}^{1+2 \epsilon+a-\alpha} \\
+\left(\left\|u_{0}\right\|_{c_{2}} \theta_{j}^{-c_{2}}+\delta \theta_{j}^{-\alpha-\epsilon}\right) \delta \theta_{j}^{-\alpha-1}\left(\left\|u_{0}\right\|_{3+2 \epsilon+a}+\delta+\delta \theta_{j}^{(a-\alpha-\epsilon)_{+}}\right) .
\end{gathered}
$$

Similarly, as the remainder of the first Taylor approximation, $e_{j}^{\prime \prime}$ is also controlled by $\Phi^{\prime \prime}$, and analogous estimates result in

$$
\begin{aligned}
\left\|e_{j}^{\prime \prime}\right\|_{2 \epsilon+a} & \lesssim \triangle_{j}\left\{\left\|\dot{u}_{j}\right\|_{2+3 \epsilon+a}\left\|\dot{u}_{j}\right\|_{0}+\left\|\dot{u}_{j}\right\|_{0}^{2}\left(\left\|u_{j}\right\|_{3+2 \epsilon+a}+\left\|\dot{u}_{j}\right\|_{3+2 \epsilon+a}\right)\right\} \\
& \lesssim \triangle_{j}\left\{\delta \theta_{j}^{1+3 \epsilon+a-\alpha} \delta \theta_{j}^{-\alpha-1}+\left(\delta \theta_{j}^{-\alpha-1}\right)^{2}\left(\left\|u_{0}\right\|_{3+2 \epsilon+a}+\delta+\delta \theta_{j}^{(3+\epsilon+a-\alpha)_{+}}\right\} .\right.
\end{aligned}
$$

To estimate $g_{m+1}$ in (45) and (46), it remains bound sums $\sum_{j=0}^{m-1} \triangle_{j}\left\{\left\|e_{j}^{\prime}\right\|_{b}+\left\|e_{j}^{\prime \prime}\right\|_{b}\right\}$. We consider a generic term of the form $\sum_{j=0}^{m-1} \triangle_{j} \theta_{j}^{-d} F\left(\delta, u_{0}\right)$ for suitable $F$ obtained from 49) resp. $\sum_{j=0}^{m-1} \triangle_{j}^{2} \theta_{j}^{-d} F\left(\delta, u_{0}\right)$ from (50). Concerning the former, if $d>1$, we have for any small $\tau>0$

$$
\sum_{j=0}^{m-1} \triangle_{j} \theta_{j}^{-d} \leq \theta_{0}^{-d+1+\tau} \sum_{j=0}^{m-1} \triangle_{j} \theta_{j}^{-1-\tau} \leq C_{\tau} \theta_{0}^{-d+1+\tau}
$$

with $C_{\tau}$ independent of $\theta_{0} \geq \theta_{0}^{\min }>0$ and $\kappa>\kappa_{\text {min }}>0$. Here we have used that

$$
\triangle_{j} \theta_{j}^{-1-\tau} \lesssim \kappa^{-1} \theta_{j}^{1-\kappa-1-\tau}=\kappa^{-1}\left(\theta_{0}^{\kappa}+j\right)^{-1-\frac{\tau}{\kappa}} .
$$

For $d<1$,

$$
\sum_{j=0}^{m-1} \triangle_{j} \theta_{j}^{-d} \leq \theta_{k}^{-d+1+\tau} \sum_{j=0}^{m-1} \triangle_{j} \theta_{j}^{-1-\tau} \leq C_{\tau} \theta_{m}^{-d+1+\tau}
$$

Finally, for $d=1$

$$
\sum_{j=0}^{m-1} \triangle_{j} \theta_{j}^{-1} \leq C_{\tau} \theta_{m}^{\tau}
$$

As for the term coming from 50

$$
\sum_{j=0}^{m-1} \triangle_{j}^{2} \theta_{j}^{-d} \leq C_{\tau} \kappa^{-1} \theta_{0}^{2-d-\kappa+\tau}
$$

Estimating the sums in (45) resp. (46) thus increases the exponent on $\theta_{m}$ resp. $\theta_{0}$ in the estimates of $e_{m}^{\prime}$ by at most $1+\tau$. From the estimates of $e_{m}^{\prime \prime}$, one obtains $\theta_{0}$ raised to a power which is arbitrarily negative for large $\kappa$. 
As a result

$$
\begin{aligned}
\theta_{m}^{a+\varepsilon-c^{\prime \prime}-1} \sum_{j=0}^{m-1} \triangle_{j}\left\|e_{j}^{\prime}\right\|_{c^{\prime \prime}} \lesssim & \delta\left\|u_{0}\right\|_{b} \theta_{m}^{a+\varepsilon-c^{\prime \prime}-1} \theta_{m / 0}^{3+\varepsilon+c^{\prime \prime}-b+\tau}+\delta^{2} \theta_{m}^{a+\varepsilon-c^{\prime \prime}-1} \theta_{m / 0}^{2+c^{\prime \prime}-2 \alpha+\tau} \\
& +\delta\left\|u_{0}\right\|_{c_{1}} \theta_{m}^{a+\varepsilon-c^{\prime \prime}-1} \theta_{m / 0}^{2-c_{1}+c^{\prime \prime}-\alpha+\tau}+\delta^{2} \theta_{m}^{a+\varepsilon-c^{\prime \prime}-1} \theta_{m / 0}^{2-\varepsilon+c^{\prime \prime}-2 \alpha+\tau} \\
& +\delta\left\|u_{0}\right\|_{c_{2}}\left\|u_{0}\right\|_{3+c^{\prime \prime}} \theta_{m}^{a+\varepsilon-c^{\prime \prime}-1} \theta_{m / 0}^{-c_{2}-\alpha+\tau} \\
& +\delta^{2}\left\|u_{0}\right\|_{3+c^{\prime \prime}} \theta_{m}^{a+\varepsilon-c^{\prime \prime}-1} \theta_{m / 0}^{-\varepsilon-2 \alpha+\tau}+\delta^{2}\left\|u_{0}\right\|_{c_{2}} \theta_{m}^{a+\varepsilon-c^{\prime \prime}-1} \theta_{m / 0}^{-c_{2}-\alpha+\tau} \\
& +\delta^{3} \theta_{m}^{a+\varepsilon-c^{\prime \prime}-1} \theta_{m / 0}^{-\varepsilon-2 \alpha+\tau}+\delta^{2}\left\|u_{0}\right\|_{c_{2}} \theta_{m}^{a+\varepsilon-c^{\prime \prime}-1} \theta_{m / 0}^{-c_{2}-\alpha+\left(c^{\prime \prime}-\alpha-3 \varepsilon\right)_{+}+\tau} \\
& +\delta^{3} \theta_{m}^{a+\varepsilon-c^{\prime \prime}-1} \theta_{m / 0}^{-\varepsilon-2 \alpha+\left(c^{\prime \prime}-\alpha-3 \varepsilon\right)_{+}+\tau}
\end{aligned}
$$

Here $\theta_{m / 0}$ is $\theta_{m}$ or $\theta_{0}$, depending on whether its exponent is greater or smaller $\tau$. Choosing e.g. $c^{\prime \prime}=\alpha+2 \varepsilon, b=3+\varepsilon+c^{\prime \prime}+2 \tau, c_{1}=2+c^{\prime \prime}-\alpha+2 \tau$ and $c_{2}=0$, the exponents of $\theta_{m / 0}$ are negative and the exponent of $\theta_{m}$ is strictly smaller than $E=a-\alpha-1$. Similarly, we obtain

$$
\begin{aligned}
\theta_{m}^{a+\varepsilon-c^{\prime \prime}-1} \sum_{j=0}^{m-1} \triangle_{j}\left\|e_{j}^{\prime \prime}\right\|_{c^{\prime \prime}} \lesssim & \delta^{2} \theta_{m}^{a+\varepsilon-c^{\prime \prime}-1} \theta_{0}^{\varepsilon+c^{\prime \prime}-2 \alpha+2-\kappa+\tau}+\delta^{2}\left(\left\|u_{0}\right\|_{3+c^{\prime \prime}}+\delta\right) \theta_{m}^{a+\varepsilon-c^{\prime \prime}-1} \theta_{0}^{-2 \alpha-\kappa+\tau} \\
& +\delta^{3} \theta_{m}^{a+\varepsilon-c^{\prime \prime}-1} \theta_{0}^{-2 \alpha-\kappa+\tau}
\end{aligned}
$$

where the exponents of the $\theta_{0}$ and $\theta_{m}$ have the same properties as in (51).

It remains to estimate the term $\theta_{m}^{\left(a+\varepsilon-c^{\prime}\right)_{+}}\left\|e_{m}\right\|_{c^{\prime}}$ in (45). We choose $c^{\prime}=a+\varepsilon$ and, in 49), set $c_{1}=c_{2}$ equal to the above $c^{\prime \prime}$ obtain

$$
\begin{aligned}
\theta_{m}^{\left(a+\varepsilon-c^{\prime}\right)_{+}}\left\|e_{m}\right\|_{c^{\prime}} \lesssim( & \left.\left\|u_{0}\right\|_{b} \theta_{m}^{2+2 \epsilon+a-b}+\delta \theta_{m}^{2+\epsilon+a-\alpha}\right) \delta \theta_{m}^{-\alpha-1}+\left(\left\|u_{0}\right\|_{c^{\prime \prime}} \theta_{m}^{-c^{\prime \prime}}+\delta \theta_{m}^{-\alpha-\epsilon}\right) \delta \theta_{m}^{1+\epsilon+a-\alpha} \\
& +\left(\left\|u_{0}\right\|_{c^{\prime \prime}} \theta_{m}^{-c^{\prime \prime}}+\delta \theta_{m}^{-\alpha-\epsilon}\right) \delta \theta_{m}^{-\alpha-1}\left(\left\|u_{0}\right\|_{3+\epsilon+a}+\delta+\delta \theta_{m}^{(a-\alpha)_{+}}\right) \\
& +\delta^{2} \triangle_{m} \theta_{m}^{2 \epsilon+a-2 \alpha}+\delta^{2} \triangle_{m} \theta_{m}^{-2 \alpha-2}\left(\left\|u_{0}\right\|_{3+\varepsilon+a}+\delta\right)+\delta^{3} \triangle_{m} \theta_{m}^{-2 \alpha-2+(3+a-\alpha)_{+}} .
\end{aligned}
$$

The exponent of $\theta_{m}$ is again strictly smaller than $E$.

The analysis of (46) is analogous. This completes the proof of Theorem 2.

\subsection{Proof of Theorem 3}

We consider the operator $A$ as an unbounded operator on the Hölder space $\mathscr{H}^{a}$ with domain $D(A)=\mathscr{H}^{a+2}$ (if $a \notin \mathbb{N}_{0}$ ). Using the nonpositivity of $A$ and [29, Theorem 9.3], we see that $A-\lambda$ is invertible for $\lambda \in \mathcal{S}_{\theta}=\{\lambda \in \mathbb{C} \backslash\{0\}:|\arg \lambda|<\theta\}, \theta \in(\pi / 2, \pi)$, and that $(A-\lambda)^{-1}$ is a pseudodifferential operator, depending on the parameter $\lambda$, whose symbol decays as $\frac{C}{|\lambda|}$. The mapping properties [30, Proposition 8.6] of such operators in Hölder spaces, which are analogous to those for Sobolev spaces, therefore imply

$$
\left\|(A-\lambda)^{-1} u\right\|_{a} \leq \frac{C}{|\lambda|}\|u\|_{a}, \quad \forall \lambda \in S_{\theta, 0} .
$$

Equation (53) allows to define the analytic semigroup generated by $A$,

$$
e^{t A} u:=\frac{1}{2 \pi i} \int_{\gamma_{r, \eta}} e^{t \lambda}(A-\lambda)^{-1} u d \lambda, \quad t>0,
$$


where $r>0, \eta \in] \pi / 2, \pi\left[\right.$, and $\gamma_{r, \eta}$ is the curve $\{\lambda \in \mathbb{C}:|\arg \lambda|=\eta,|\lambda| \geq r\} \cup\{\lambda \in \mathbb{C}:|\arg \lambda| \leq$ $\eta,|\lambda|=r\}$, oriented counterclockwise. $e^{t A} u$ does not depend on the choice of $r$ and $\eta$. We recall some basic properties of analytic semigroups (Proposition 2.1.1, [31]):

Proposition 2. (i) $\left\|e^{t A} u\right\|_{a} \leq C_{0}\|u\|_{a}, \quad \forall t \geq 0$.

(ii) $e^{t A} e^{s A}=e^{(t+s) A}, \quad \forall t, s \geq 0$.

(iii) $\lim _{t \rightarrow 0^{+}}\left\|e^{t A} u-u\right\|_{a}=0, \quad \forall u \in \overline{D(A)}$.

(iv) There are constants $C_{l}$, such that

$$
\left\|t^{l} A^{l} e^{t A} u\right\|_{a} \leq C_{l}\|u\|_{a}, \quad 0<t \leq 1 .
$$

(v) $t \mapsto e^{t A}$ is a real-analytic function from $(0, \infty)$ to the Banach space of bounded linear operators on $\mathcal{H}^{a}$ (with norm given by the operator norm) and

$$
\frac{d^{l}}{d t^{l}} e^{t A}=A^{l} e^{t A}, \quad t>0 .
$$

Concerning Theorem 3, we first consider Property (0). Using Proposition 2(iii) and setting $S_{\theta}=e^{t A}$ and $t=\theta^{-2 k}$ we have

$$
\lim _{\theta \rightarrow \infty} S_{\theta} u=u, \quad \forall u \in \overline{\mathscr{H}^{2+a}}
$$

and thus, Property (0) holds.

Using Proposition 2 (i) and the fact that $S_{\theta}=e^{t A}$ is a continuous operator on $\mathscr{H}^{b}$ we have

$$
\left\|e^{t A} u\right\|_{b} \lesssim\|u\|_{b} \lesssim\|u\|_{a}, \quad \forall b \leq a
$$

and thus also Property (i).

In order to prove Property (ii), note that it suffices to show the assertion for $0<t \leq 1$, or equivalently $\theta \geq 1$. We use that $(A-1)^{-1}: \mathscr{H}^{a} \rightarrow \mathscr{H}^{a+2 k}$ is continuous, $\left\|(A-1)^{-1} u\right\|_{\mathscr{H}^{a+2 k}} \lesssim$ $\|u\|_{\mathscr{H}^{a}}$. We then have

$$
\|v\|_{a+2 k} \lesssim\|(A-1) v\|_{a} \lesssim\|A v\|_{a}+\|v\|_{a} .
$$

We first set $l=1$ and $v=t e^{t A} u$ and deduce

$$
\left\|t e^{t A} u\right\|_{a+2 k} \lesssim\left\|(A-1) t e^{t A} u\right\|_{a} \lesssim\left\|t A e^{t A} u\right\|_{a}+\left\|t e^{t A} u\right\|_{a}
$$

and by using Proposition 2(i) and Proposition 2(iv) we have

$$
\left\|t e^{t A} u\right\|_{a} \leq\left\|e^{t A} u\right\|_{a} \lesssim\|u\|_{a}, \quad 0<t \leq 1
$$

and finally by (58) we obtain

$$
\left\|t e^{t A} u\right\|_{a+2 k} \lesssim\|u\|_{a} .
$$

By iterating this argument $l$-times using

$$
\left\|t^{l} e^{t A} u\right\|_{a+2 k l}=l^{l}\left\|\frac{t}{l} e^{t / l} A \cdot \ldots \cdot \frac{t}{l} e^{t / l} A u\right\|_{a+2 k l}
$$


we have

$$
\left\|t^{l} e^{t A} u\right\|_{a+2 k l} \lesssim\|u\|_{a} .
$$

Setting $b=a+2 k l, t=\theta^{-2 k}$, Property (ii) holds for this specific $b$.

For an arbitrary $b, \tilde{b}:=a+2 k l \geq b$, write $b=\sigma a+(1-\sigma) \tilde{b}$. The interpolation estimate (27) gives

$$
\left\|e^{t A} u\right\|_{b} \leq\left\|e^{t A} u\right\|_{a}^{\lambda}\left\|e^{t A} u\right\|_{\tilde{b}}^{1-\lambda} \lesssim t^{-l(1-\lambda)}\|u\|_{a}^{\lambda}\|u\|_{a}^{1-\lambda}
$$

and we deduce

$$
\left\|e^{t A} u\right\|_{b} \lesssim t^{-(1-\lambda) l}\|u\|_{a}=t^{-(b-a) / 2 k}\|u\|_{a} .
$$

Setting now $S_{\theta}:=e^{t A}$ with $t=\theta^{-2 k}$ we have proved

$$
\left\|S_{\theta} u\right\|_{b} \lesssim \theta^{b-a}\|u\|_{a}
$$

and thus, Property (ii) holds.

For Property (iv) we first use $t=\theta^{-2 k}$ and observe

$$
\frac{d}{d \theta} e^{t A} u=\frac{d t}{d \theta} \frac{d}{d t} e^{t A} u=-2 k t^{1 / 2 k}\left(t A e^{t A} u\right)=-\frac{2 k}{\theta} t A e^{t A} u .
$$

The same proof as for Property (ii) yields, setting $S_{\theta}=e^{t A}$ :

$$
\left\|\frac{d}{d \theta} S_{\theta} u\right\|_{b}=\frac{2 k}{\theta}\left\|t A e^{t A} u\right\|_{b} \lesssim \frac{2 k}{\theta} \theta^{b-a}\|u\|_{a}=2 k \theta^{b-a-1}\|u\|_{a} .
$$

Finally, given the continuity of $S_{\theta}$ on $\mathscr{H}^{a}$, it suffices to show Property $\left(i i i^{\prime}\right)$ for $b \neq a$. Note that $1-A: \mathscr{H}^{a+2 k} \rightarrow \mathscr{H}^{a}$ is an isomorphism. With $1-e^{t \lambda}=-\lambda \int_{0}^{t} e^{\lambda s} d s$, we have for $u \in C^{\infty}$, $v=(1-A)^{\frac{a-b}{2 k}} u$ and $t \in(0,1]$

$$
\begin{aligned}
u-e^{t A} u & =\frac{1}{2 \pi i} \int_{\gamma_{r, \eta}}\left(1-e^{t \lambda}\right)(A-\lambda)^{-1}(1-A)^{-\frac{a-b}{2 k}} v d \lambda \\
& =-\frac{1}{2 \pi i} \int_{\gamma_{r, \eta}} \lambda \int_{0}^{t} e^{\lambda s} d s(A-\lambda)^{-1}(1-\lambda)^{-\frac{a-b}{2 k}} v d \lambda .
\end{aligned}
$$

The double integral is absolutely convergent for $\frac{a-b}{2 k} \in(0,1)$. After interchanging the order of integration and using the triangle inequality as well as $\left\|(A-\lambda)^{-1} v\right\|_{b} \lesssim \frac{\|v\|_{b}}{\lambda}$, the right hand side is smaller than a constant times

$$
\int_{0}^{t} \int_{\gamma_{r, \eta}} e^{s \operatorname{Re} \lambda}|1-\lambda|^{-\frac{a-b}{2 k}}\|v\|_{b}|d \lambda| d s .
$$

If $r<1$, we may bound $|1-\lambda|^{-1} \leq C_{r}(1+|\lambda|)^{-1}$ for all $\lambda \in \gamma_{r, \eta}$. It therefore remains to estimate

$$
\int_{0}^{t} \int_{\gamma_{r, \eta}} e^{s \operatorname{Re} \lambda}(1+|\lambda|)^{-\frac{a-b}{2 k}}\|v\|_{b}|d \lambda| d s .
$$

We split the integral $\int_{\gamma_{r, \eta}}=I_{r}+I_{+}+I_{-}$into integrals over $\gamma_{r}=\left\{\lambda=r e^{i \sigma} \in \mathbb{C}:|\sigma| \leq \eta\right\}$, $\gamma_{+}=\left\{\lambda=\rho e^{i \eta} \in \mathbb{C}: \rho \geq r\right\}$ resp. $\gamma_{-}=\left\{\lambda=\rho e^{-i \eta} \in \mathbb{C}: \rho \geq r\right\}$ and consider the three terms separately. The first integral,

$$
I_{r}=\int_{0}^{t} \int_{-\eta}^{\eta} e^{s \cos (\sigma)} d \sigma d s(1+r)^{-\frac{a-b}{2 k}}\|v\|_{b}
$$


is bounded by $t(2 \eta) e(1+r)^{-\frac{a-b}{2 k}}\|v\|_{b}$ and hence of order $t$. For the second and third integrals,

$$
I_{ \pm}=\int_{0}^{t} \int_{r}^{\infty} e^{-s \rho|\cos (\eta)|}(1+\rho)^{-\frac{a-b}{2 k}}\|v\|_{b} d \rho d s
$$

the change of variables $\rho \mapsto \frac{\rho}{s|\cos (\eta)|}$ leads to

$$
I_{ \pm}=\int_{0}^{t} \int_{s r|\cos (\eta)|}^{\infty} e^{-\rho}\left(\frac{s|\cos (\eta)|}{s|\cos (\eta)|+\rho}\right)^{\frac{a-b}{2 k}}\|v\|_{b} \frac{1}{s|\cos (\eta)|} d \rho d s
$$

or

$$
I_{ \pm} \leq \int_{0}^{t}(s|\cos (\eta)|)^{\frac{a-b}{2 k}-1} \int_{0}^{\infty} e^{-\rho} \rho^{-\frac{a-b}{2 k}}\|v\|_{b} d \rho d s \lesssim t^{\frac{a-b}{2 k}}\|v\|_{b}
$$

Using $t=\frac{1}{\theta^{2 k}}$ and $\|v\|_{b}=\left\|(1-A)^{\frac{a-b}{2 k}} u\right\|_{b} \lesssim\|u\|_{a}$, (iii') follows.

\section{Acknowledgments}

We thank Lothar Banz for instructive discussions about computational aspects of this work. This work was supported by the cluster of excellence QUEST, the Danish National Research Foundation (DNRF) through the Centre for Symmetry and Deformation and the Danish Science Foundation (FNU) through research grant 10-082866. H. G. thanks the Institut für Angewandte Mathematik for hospitality.

\section{References}

[1] B. Heck. Integral equation methods in physical geodesy. In Grafarend, E.W./Krumm, F.W./Schwarze,V.S. (Eds.):Geodesy - The Challenge of the 3rd Millenium, pages 197-206. Springer, 2002.

[2] W. A. Heiskanen and H. Moritz. Physical Geodesy. W. H. Freeman, San Francisco, 1993 reprint edition, 1967.

[3] M.S. Molodenskii. Grundbegriffe der geodätischen Gravimetrie. VEB Verlag Technik, Berlin, 1958.

[4] M.S. Molodenskii. Methods for study of the external gravitational field and figure of the earth. Jerusalem, Israel Program for Scientific Translations; [available from the Office of Technical Services, U.S. Dept. of Commerce, Washington], 1962.

[5] L. Hörmander. The boundary problems of physical geodesy. Arch. Rational Mech. Anal., 62(1):1-52, 1976.

[6] J. Nash. The imbedding problem for Riemannian manifolds. Ann. of Math. (2), 63:20-63, 1956.

[7] J. Moser. A new technique for the construction of solutions of nonlinear differential equations. Proc. Nat. Acad. Sci. U.S.A., 47:1824-1831, 1961. 
[8] A. A. Ardalan and E. W. Grafarend. Somiglianapizzetti gravity: the international gravity formula accurate to the sub-nanogal level. Journal of Geodesy, 75:424-437, 2001.

[9] R. Klees, M. van Gelderen, C. Lage, and C. Schwab. Fast numerical solution of the linearized Molodensky problem. Journal of Geodesy, 75:349-362, 2001. 10.1007/s001900100183.

[10] P. Holota. Coerciveness of the linear gravimetric boundary problem and a geometrical interpretation. Journal of Geodesy, 71:640-651, 1997.

[11] W. Freeden and C. Mayer. Multiscale solution for the Molodensky problem on regular telluroidal surfaces. Acta Geodaetica et Geophysica Hungarica, 41:55-86, 2006.

[12] J. W. Jerome. An adaptive Newton algorithm based on numerical inversion: regularization as postconditioner. Numer. Math., 47(1):123-138, 1985.

[13] G. Fasshauer and J. W. Jerome. Multistep approximation algorithms: Improved convergence rates through postconditioning with smoothing kernels. Advances in Computational Mathematics, 10:1-27, 1999. 10.1023/A:1018962112170.

[14] H. Wendland. A high-order approximation method for semilinear parabolic equations on spheres. Math. Comp., page to appear, 2012.

[15] T. Krarup. Letters on Molodensky's problem, iii. Unpublished manuscript, Geodaetisk Institut, 1973.

[16] A. Schlömerkemper. About solutions of Poisson's equation with transition condition in nonsmooth domains. Z. Anal. Anwend., 27(3):253-281, 2008.

[17] C. Schwab. Variable order composite quadrature of singular and nearly singular integrals. Computing, 53(2):173-194, 1994.

[18] S. A. Sauter and C. Schwab. Boundary Element Methods, volume 39 of Springer Series in Computational Mathematics. Springer Verlag, Berlin, 2011.

[19] Tran T. Stephan, E.P. and A. Costea. A boundary integral equation on the sphere for high-precision geodesy. Computer Methods in Mechanics: Lectures of the CMM 2009, pages 99-110, 2010.

[20] A. Costea. Mathematical modelling and numerical simulations in physical geodesy. Dissertation, Leibniz University Hannover, 2012.

[21] R. Seeley. Topics in pseudo-differential operators. In C.I.M.E. Conference on pseudodifferential operators, Stresa 1968, pages 167-305. Cremonese, Rome, 1969.

[22] S. Seo, M. K. Chung, and H. K. Vorperian. Heat kernel smoothing using Laplace-Beltrami eigenfunctions. In Proceedings of the 13th international conference on Medical image computing and computer-assisted intervention: Part III, MICCAI'10, pages 505-512, Berlin, Heidelberg, 2010. Springer-Verlag. 
[23] M. Maischak. Technical manual of the program system maiprogs, 2001.

[24] M. Maischak. Analytical evaluation of potentials and computation of galerkin integrals on triangles and parallelograms. Technical report, ifam50, 2001.

[25] S. Erichsen and S. A. Sauter. Efficient automatic quadrature in 3-d Galerkin BEM. Comput. Methods Appl. Mech. Engrg., 157(3-4):215-224, 1998. Seventh Conference on Numerical Methods and Computational Mechanics in Science and Engineering (NMCM 96) (Miskolc).

[26] C. Schwab and W. Wendland. On the extraction technique in boundary integral equations. Math. Comp., 68(225):91-122, 1999.

[27] H. Schulz, C. Schwab, and W. L. Wendland. The computation of potentials near and on the boundary by an extraction technique for boundary element methods. Comput. Methods Appl. Mech. Engrg., 157(3-4):225-238, 1998. Seventh Conference on Numerical Methods and Computational Mechanics in Science and Engineering (NMCM 96) (Miskolc).

[28] J.-C. Nédélec. Acoustic and Electromagnetic Equations. Springer-Verlag, New York, 2000.

[29] M. A. Shubin. Pseudodifferential operators and spectral theory. Springer-Verlag, Berlin, second edition, 2001. Translated from the 1978 Russian original by Stig I. Andersson.

[30] M. E. Taylor. Partial differential equations III. Nonlinear equations, volume 117 of Applied Mathematical Sciences. Springer, New York, second edition, 2011.

[31] A. Lunardi. Analytic semigroups and optimal regularity in parabolic problems. Progress in Nonlinear Differential Equations and their Applications, 16. Birkhäuser Verlag, Basel, 1995. 